\title{
A SPLITTING METHOD FOR THE AUGMENTED BURGERS EQUATION
}

\begin{abstract}
In this paper we consider a splitting method for the augmented Burgers equation and prove that it is of first order. We also analyze the large-time behavior of the approximated solution by obtaining the first term in the asymptotic expansion. We prove that, when time increases, these solutions behave as the self-similar solutions of the viscous Burgers equation.
\end{abstract}

\section{INTRODUCTION}

In this paper we consider a splitting method for the following augmented Burgers equation:

$$
\begin{cases}u_{t}-\left(\frac{u^{2}}{2}\right)_{x}=u_{x x}+K * u-u+u_{x}, & (t, x) \in(0, \infty) \times \mathbb{R}, \\ u(0, x)=u_{0}(x), & x \in \mathbb{R},\end{cases}
$$

where $K(z)=e^{-z} \chi_{(0, \infty)}$. This model has been introduced in the context of the analysis of the sonicboom phenomenon to reproduce the propagation of the sound waves produced by supersonic aircrafts from their near-field down to the ground level $[9,23,24]$.

New trends in aerospace engineering have started to use nonlinear physical models for the characterization of this phenomenon, in order to be capable of building efficient supersonic civilian airplanes that can avoid such strident noise. We refer the reader to [2] for a detailed survey on the topic. In this paper we focus on the augmented Burgers equation, which was initially developed by Cleveland [9] and later adopted by Rallabhandi [23, 24]. In particular, (1.1) follows from considering constant atmosphere conditions and a single relaxation phenomenon.

Industrial applications like the aforementioned sonic-boom phenomenon, in which approximate solutions for large times are required, need a cautious numerical treatment. A good understanding of the behavior of the solutions in these extended regimes, both in continuous and discrete settings, is necessary in order to be able to simulate them accurately. In that sense, we have already analyzed equation (1.1) in [16]. There we obtain the asymptotic profile of the solution of (1.1) as time goes to infinity. Moreover, we propose a semi-discrete scheme that is capable of mimicking the large-time dynamics of the continuous system. This issue needs to be handled carefully: numerical schemes with an acceptable accuracy in short-time intervals can evolve solutions towards incorrect large-time profiles when the numerical viscosity dominates the physical one [17]. However, solving (1.1) by discretizing the convolution can be computationally expensive. In this paper we set up the framework to empower the use of splitting methods to solve the equation more efficiently. Let us mention that these techniques have already been used in $[9,23,24]$ in the context of the sonic-boom phenomenon.

Date: July 12, 2017

L. I. Ignat was partially supported by Grant PN-II-RU-TE-2014-4-0007 of the Romanian National Authority for Scientific Research and Innovation, CNCS-UEFISCDI, Grant MTM2014-52347, MICINN, Spain and FA9550-15-1-0027 of AFOSR. A. Pozo was granted by the Basque Government, reference PRE_2013_2_150, and partially supported by ERCEA under Grant 246775 NUMERIWAVES, by the Basque Government through the BERC 2014-2017 program and by Spanish MINECO: BCAM Severo Ochoa excellence accreditation SEV-2013-0323. 
Various versions of this method have also been developed, for instance, for the nonlinear Schrödinger equation, the Korteweg-de Vries equation, the Boltzmann equation... (see [13] and the references therein) and, more recently, the nonlocal Fowler equation [6].

The basic idea behind operator splitting methods is that the overall evolution operator can be formally written as a sum of evolution operators for each term appearing in the model. In other words, one separates the complete system into a set of simpler sub-equations, for which more practical algorithms are available. Once we successively solve these sub-equations, their solutions are put together to compute the complete solution of the model. We refer to [13] for a more detailed introduction on operator splitting methods.

Let us remark that the analysis performed in this paper can be extended to any kernel $K \in$ $L^{1}\left(\mathbb{R}, 1+|x|^{2}\right)$ by replacing the term $K * u-u+u_{x}$ adequately. The analysis we present here for $K(z)=e^{-z} \chi_{(0, \infty)}$ is the same for any kernel having the mass and the first moment equal to one:

$$
\int_{\mathbb{R}} K(z) d z=\int_{\mathbb{R}} K(z) z d z=1 .
$$

We introduce the following Trotter formula for the augmented Burgers equation (1.1). Let $X^{t}$ be the evolution operator associated with

$$
\begin{cases}v_{t}=K * v-v+v_{x}, & (t, x) \in(0, \infty) \times \mathbb{R}, \\ v(0, x)=v_{0}(x), & x \in \mathbb{R},\end{cases}
$$

and $Y^{t}$ the one corresponding to

$$
\begin{cases}w_{t}-\left(\frac{w^{2}}{2}\right)_{x}=w_{x x}, & (t, x) \in(0, \infty) \times \mathbb{R}, \\ w(0, x)=w_{0}(x), & x \in \mathbb{R} .\end{cases}
$$

We consider the flow $Z^{t}$ defined by

$$
Z^{t}=X^{t} Y^{t}
$$

The aim is to approximate the flow $S^{t}$ given by (1.1) by alternating both operators; that is, for a small $\Delta t>0$ and a positive integer $n$, we want to analyze whether

$$
S^{n \Delta t} u_{0}=u(n \Delta t) \approx\left(Z^{\Delta t}\right)^{n} u_{0}=\left(X^{\Delta t} Y^{\Delta t}\right)^{n} u_{0} .
$$

Subsequently we denote $Z^{n \Delta t}$ instead of $\left(Z^{\Delta t}\right)^{n}$ for the sake of clarity of the notation.

The first result of this paper is given in the following theorem. It confirms that the splitting (1.5) is first-order accurate for $H^{2}(\mathbb{R})$ initial data. For practical issues, we also consider the case of $H^{1}(\mathbb{R})$ initial data.

Theorem 1.1. Let $r \in\{1,2\}$. For any $u_{0} \in H^{r}(\mathbb{R})$ and for all $T>0$, the following hold:

$$
\left\|Z^{n \Delta t} u_{0}-u(n \Delta t)\right\|_{L^{2}(\mathbb{R})} \leq C\left(T,\left\|u_{0}\right\|_{H^{1}}\right)\left\|u_{0}\right\|_{H^{r}(\mathbb{R})}^{2}(\Delta t)^{r / 2}
$$

and

$$
\left\|Z^{n \Delta t} u_{0}\right\|_{H^{r}(\mathbb{R})} \leq C\left(T,\left\|u_{0}\right\|_{L^{2}(\mathbb{R})}\right)\left\|u_{0}\right\|_{H^{r}(\mathbb{R})},
$$

for all $\Delta t \in(0,1)$ and for all $n \in \mathbb{N}$ such that $0 \leq n \Delta t \leq T$. 
The second result of this work concerns the first term in the large time asymptotic expansion of the approximate solution $Z^{n \Delta t} u_{0}$. To be more precise, let us define time nodes $t_{n}=n \Delta t$ and $t_{n+1 / 2}=$ $\left(n+\frac{1}{2}\right) \Delta t$ for every $n \in \mathbb{N} \cup\{0\}$ and the function $u^{\Delta}$ by

$$
u^{\Delta}(t, x)= \begin{cases}Y^{2\left(t-t_{n}\right)} Z^{n \Delta t} u_{0}(x), & t \in\left[t_{n}, t_{n+1 / 2}\right), x \in \mathbb{R}, \\ X^{2\left(t-t_{n+1 / 2}\right)} Y^{\Delta t} Z^{n \Delta t} u_{0}(x), & t \in\left[t_{n+1 / 2}, t_{n+1}\right), x \in \mathbb{R} .\end{cases}
$$

This way we extend the discrete solution to a continuous-in-time framework. In particular, observe that for any $n \geq 0, u^{\Delta}\left(t_{n}\right)=Z^{n \Delta t} u_{0}$.

By construction and the properties of $X^{t}$ and $Y^{t}$, we will have that for any $u_{0} \in L^{1}(\mathbb{R}) \cap L^{\infty}(\mathbb{R})$, function $u^{\Delta}$ defined by (1.6) satisfies $u^{\Delta} \in C\left([0, \infty) ; L^{p}(\mathbb{R})\right)$ for any $1 \leq p \leq \infty$. Moreover, classical bootstrap arguments for convection-diffusion problems [12] and the properties of flow $X^{t}$ in Lemma 2.1 show that for any $1<p<\infty$ function $u^{\Delta}$ satisfies

$$
u^{\Delta} \in C\left((0, \infty) \backslash \Delta ; W^{2, p}(\mathbb{R})\right) \cap C^{1}\left((0, \infty) \backslash \Delta ; L^{p}(\mathbb{R})\right),
$$

where $\Delta=\left\{t_{n+1}, t_{n+1 / 2}, n \geq 0\right\}$.

The behavior of $u^{\Delta}$ as $t \rightarrow \infty$, so the one of $Z^{n \Delta t} u_{0}$, follows from a scaling argument. Indeed, the following result confirms that the Trotter formula (1.5) for the augmented Burgers equation (1.1) preserves the large-time behavior obtained in [16] for $u$.

Theorem 1.2. For any $u_{0} \in L^{1}(\mathbb{R}) \cap L^{\infty}(\mathbb{R})$ and for all $p \in[1, \infty)$, it holds that

$$
t^{\frac{1}{2}\left(1-\frac{1}{p}\right)}\left\|u^{\Delta}(t)-u_{M}(t)\right\|_{L^{p}(\mathbb{R})} \rightarrow 0, \quad \text { as } t \rightarrow \infty,
$$

where $u_{M}(t, x)=t^{-1 / 2} f_{M}(x / \sqrt{t})$ is the self-similar profile of the following viscous Burgers equation

$$
\begin{cases}u_{t}=\left(\frac{u^{2}}{2}\right)_{x}+2 u_{x x}, & (t, x) \in(0, \infty) \times \mathbb{R}, \\ u(0, x)=M \delta_{0}, & x \in \mathbb{R},\end{cases}
$$

where $M=\int_{\mathbb{R}} u_{0}(x) d x$ is the mass of the initial data $u_{0}$.

Let us recall, see for example [1], that equation (1.9) has a unique solution of the form $u(t, x)=$ $t^{-1 / 2} f_{M}(x / \sqrt{t})$ where the profile $f_{M}$ is smooth, decays as $|x| \rightarrow \infty$ and has mass $M$ and constant sign.

Moreover, the above theorem provides information about the asymptotic behavior of the approximation $Z^{n \Delta t}$, since $u^{\Delta}(n \Delta t)=Z^{n \Delta t} u_{0}$. For any fixed $\Delta t$ and any $1 \leq p<\infty$ we have

$$
(n \Delta t)^{\frac{1}{2}\left(1-\frac{1}{p}\right)}\left\|Z^{n \Delta t} u_{0}-u_{M}(n \Delta t)\right\|_{L^{p}(\mathbb{R})} \rightarrow 0, \quad \text { as } n \rightarrow \infty .
$$

Remark 1. In fact, the result in Theorem 1.2 is even more general, allowing big time steps. Let $\left\{\Delta t_{n}\right\}_{n \geq 1}$ a sequence of positive time steps such that $n \Delta t_{n} \rightarrow \infty$ as $n \rightarrow \infty$. Then

$$
\left(n \Delta t_{n}\right)^{\frac{1}{2}\left(1-\frac{1}{p}\right)}\left\|Z^{n \Delta t_{n}} u_{0}-u_{M}\left(n \Delta t_{n}\right)\right\|_{L^{p}(\mathbb{R})} \rightarrow 0, \text { as } n \rightarrow \infty .
$$

The proof of the above result is similar to the one in Theorem 1.2. To be more precise, the estimates in Section 3.1 and Section 3.2 are uniform with respect to the time step discretization $\Delta t_{n}$. After these steps, we consider a sequence $\left\{\lambda_{n}\right\}_{n \geq 1}$ satisfying $\lambda_{n} \geq 1, \lambda_{n} \rightarrow \infty$ such that $\Delta t_{n} / \lambda_{n}^{2} \rightarrow 0$ when $n \rightarrow \infty$. All the arguments in Section 3.3 and Section 3.4 hold under these assumption on the sequence $\left\{\lambda_{n}\right\}_{n \geq 1}$. Finally, choosing a convenient sequence $\lambda_{n}$ we obtain (1.10). 
Remark 2. Note that the constant in front of the second derivative of $u$ in (1.9) is the sum of the coefficient of $u_{x x}$ already appearing in (1.1) plus one half of the second moment of function $K$. For a general kernel $K \in L^{1}\left(\mathbb{R}, 1+x^{2}\right)$, the splitting approximation of the solution of the equation

$$
\left\{\begin{array}{l}
u_{t}=\left(\frac{u^{2}}{2}\right)_{x}+u_{x x}+\int_{\mathbb{R}} K(x-y)\left(u(y)-u(x)-(y-x) u_{x}(x)\right) d y, \\
u(0, x)=u_{0}(x),
\end{array}\right.
$$

behaves for large time as the solution of the system

$$
\left\{\begin{array}{l}
u_{t}=\left(\frac{u^{2}}{2}\right)_{x}+\left(1+\frac{M_{2}}{2}\right) u_{x x}, \\
w(0)=M \delta_{0}
\end{array}\right.
$$

where $M_{2}$ is the second moment of the kernel $K$.

The analysis of the long time behavior of nonlinear evolution problems with nonlocal diffusion has been considered in various papers. For example, we refer the reader to $[15,18,19,22]$ for smooth kernels and to $[3,4,5,20]$ for singular kernels of Lévy type. We have adapted to the numerical level some of the ideas from these papers in order to prove Theorem 1.2.

The rest of the paper is organized as follows. In Section 2 we show the convergence of the approximation $Z^{n \Delta t} u_{0}$ obtained by means of the splitting method, as well as its accuracy order. Then, in Section 3, we obtain the decay estimates of the $L^{p}$-norms of $u^{\Delta}$ in (1.6). We use them, together with a scaling argument, to prove Theorem 1.2. We conclude with some numerical experiments that illustrate our results in Section 4.

\section{CONVERGENCE OF THE SPLitTing METHOD}

This section is devoted to the proof of Theorem 1.1. We first obtain some preliminary results on the flows $X^{t}$ and $Y^{t}$. We also consider the flow $S^{t}$ and estimate it in the Sobolev spaces $H^{s}(\mathbb{R})$. These allow us to obtain local error estimates for flow $Z^{t}$, which lead us to establish the order of convergence of the splitting method.

2.1. Stability of the flows $X^{t}$ and $Y^{t}$. Let us denote by $D_{t}$ the kernel of the semigroup corresponding to (1.3). It follows that $X^{t} v_{0}=D_{t} * v_{0}$. We first prove that the flow $X^{t}$ is stable both in $L^{p}(\mathbb{R})$ and $H^{s}(\mathbb{R})$ for any $1 \leq p \leq \infty$ and $s \geq 0$. Then, we obtain local error estimates for $X^{t}$.

Lemma 2.1. The flux $X^{t}$ corresponding to (1.3) has the following properties:

(1) For any $v_{0}, v_{1} \in L^{1}(\mathbb{R})$ satisfying $v_{0} \leq v_{1}$, we have

$$
X^{t} v_{0} \leq X^{t} v_{1}, \quad \forall t \geq 0 .
$$

(2) For any $p \geq 1$ and $v_{0} \in L^{p}(\mathbb{R})$, it holds that

$$
\left\|X^{t} v_{0}\right\|_{L^{p}(\mathbb{R})} \leq\left\|v_{0}\right\|_{L^{p}(\mathbb{R})}, \quad \forall t \geq 0 .
$$

(3) For any $s \geq 0$ and $v_{0} \in H^{s}(\mathbb{R})$, it holds that

$$
\left\|X^{t} v_{0}\right\|_{H^{s}(\mathbb{R})} \leq\left\|v_{0}\right\|_{H^{s}(\mathbb{R})}, \quad \forall t \geq 0 .
$$

(4) For any integer $n \geq 1$, any real number $p \geq 1$ and $v_{0} \in W^{n, p}(\mathbb{R})$, it holds that

$$
\left\|X^{t} v_{0}\right\|_{W^{n, p}(\mathbb{R})} \leq\left\|v_{0}\right\|_{W^{n, p}(\mathbb{R})}, \quad \forall t \geq 0 .
$$


Proof. We proceed in a classical way. Even if this is by now a well known method (see, for example, the proof of Prop. 1 in [12] or [11, Ch. VI]), we will try to make the details clear for the convenience of the reader. In the case of the first property, we can consider smooth solutions, i.e. of Schwartz class, and use that for any function $\varphi \in C(\mathbb{R})$ the following holds

$$
\int_{\mathbb{R}} v_{x}(t, x) \varphi(v(t, x)) d x=0, \quad \forall t>0 .
$$

By an extension argument, this identity may be extended to $\varphi(s)=\operatorname{sign}(s), \varphi(s)=s^{+}$or $\varphi(s)=s^{-}$, where $z^{+}:=\max \{0, z\}$ and $z^{-}:=\min \{0, z\}$. Moreover, it is sufficient to consider the case $v_{1} \equiv 0$ and prove that

$$
\frac{d}{d t} \int_{\mathbb{R}} v^{+}(t, x) d x=\int_{\mathbb{R}}\left(K * v-v+v_{x}\right)(t, x) \operatorname{sign}\left(v^{+}(t, x)\right) d x \leq 0 .
$$

Using that for any $x, y \in \mathbb{R}$ we have

$$
v(t, y) \operatorname{sign}\left(v^{+}(t, x)\right) \leq v^{+}(t, y),
$$

we obtain

$$
\begin{aligned}
\int_{\mathbb{R}}(K * v)(t, x) \operatorname{sign}\left(v^{+}(t, x)\right) d x & =\int_{\mathbb{R}} \int_{\mathbb{R}} K(x-y) v(t, y) \operatorname{sign}\left(v^{+}(t, x)\right) d x d y \\
\leq \int_{\mathbb{R}} \int_{\mathbb{R}} K(x-y) v^{+}(t, y) d x d y & =\int_{\mathbb{R}} v^{+}(t, y) d y
\end{aligned}
$$

and the desired comparison principle is proved.

Let us focus now on the second property. For $p=1$ we multiply equation $(1.3)$ by $\operatorname{sign}(v)$ and integrate it on the whole space. Since $K$ has mass one, we obtain that the $L^{1}(\mathbb{R})$-norm of $v$ decreases in time:

$$
\frac{d}{d t} \int_{\mathbb{R}}|v(t, x)| d x=\int_{\mathbb{R}}\left(K * v-v+v_{x}\right) \operatorname{sign}(v) \leq 0 .
$$

In the case $p=\infty$, we multiply by $\operatorname{sign}(v-\mu)^{+}$, where $\mu=\left\|v_{0}\right\|_{L^{\infty}(\mathbb{R})}$, and apply the same strategy. For a rigorous justification of the above argument we can use the same procedure as in the case of the first property.

Regarding the third property, using the Fourier transform, we have that

$$
\widehat{K}(\xi)=\frac{1}{1+i \xi}, \xi \in \mathbb{R}
$$

and

$$
\widehat{D_{t}}(\xi)=e^{t(\widehat{K}(\xi)-1+i \xi)}=\exp \left(\frac{-t \xi^{2}}{1+i \xi}\right), \xi \in \mathbb{R}
$$

Thus, it is enough to observe that $\left|\widehat{D_{t}}(\xi)\right| \leq 1$.

For the last property it is sufficient to observe that $\partial_{x}$ commutes with $X^{t}$ and to use the second property.

Lemma 2.2 (Local error estimates for $X^{t}$ ). For any $v_{0} \in H^{1}(\mathbb{R})$ the following holds:

$$
\left\|\left(X^{t}-I\right) v_{0}\right\|_{L^{2}(\mathbb{R})} \leq t\left\|\partial_{x} v_{0}\right\|_{L^{2}(\mathbb{R})}, \quad \forall t \geq 0 .
$$


Proof. From (1.3), we obtain that

$$
\left\|\left(X^{t}-I\right) v_{0}\right\|_{L^{2}(\mathbb{R})} \leq \int_{0}^{t}\left\|K * v(\tau)-v(\tau)+v_{x}(\tau)\right\|_{L^{2}(\mathbb{R})} d \tau .
$$

Using Plancherel's identity and (2.2) we have, for any $\tau \geq 0$,

$$
\begin{gathered}
\left\|K * v(\tau)-v(\tau)+v_{x}(\tau)\right\|_{L^{2}(\mathbb{R})}^{2}=\int_{\mathbb{R}}\left|\frac{-\xi^{2}}{1+i \xi}\right|^{2}|\widehat{v}(\tau, \xi)|^{2} d \xi=\int_{\mathbb{R}} \frac{\xi^{4}}{1+\xi^{2}}|\widehat{v}(\tau, \xi)|^{2} d \xi \\
\leq \int_{\mathbb{R}} \xi^{2}\left|\exp \left(\frac{-\tau \xi^{2}}{1+i \xi}\right)\right|^{2}\left|\widehat{v_{0}}(\xi)\right|^{2} d \xi \leq\left\|\partial_{x} v_{0}\right\|_{L^{2}(\mathbb{R})}^{2} .
\end{gathered}
$$

Therefore, we deduce the desired estimate.

Let us now focus on the flow $Y^{t}$, which corresponds to the well-known viscous Burgers equation. For the sake of completeness, we recall the following estimates.

Lemma 2.3. The flux $Y^{t}$ corresponding to (1.4) has the following properties:

(1) For any $w_{0}, w_{1} \in L^{1}(\mathbb{R})$ such that $w_{0} \leq w_{1}$, we have

$$
Y^{t} w_{0} \leq Y^{t} w_{1}, \quad \forall t \geq 0 .
$$

(2) For any $p \geq 1$ and $w_{0} \in L^{p}(\mathbb{R})$, it holds that

$$
\left\|Y^{t} w_{0}\right\|_{L^{p}(\mathbb{R})} \leq\left\|w_{0}\right\|_{L^{p}(\mathbb{R})}, \quad \forall t \geq 0 .
$$

(3) Let $n \in \mathbb{N}$ and $w_{0} \in H^{n}(\mathbb{R})$. If $\left\|w_{0}\right\|_{L^{2}(\mathbb{R})} \leq M$, then for any $T>0$ there exists $C=C(T, M)$ such that

$$
\left\|Y^{t} w_{0}\right\|_{H^{n}(\mathbb{R})} \leq e^{C t}\left\|w_{0}\right\|_{H^{n}(\mathbb{R})}, \quad \forall t \in[0, T]
$$

Proof. The first property is classical in the context of viscous approximations of conservation laws. We refer to [11, Th. 6.3.2, p. 184] for a detailed proof.

The second one follows from classical estimates for convection-diffusion equations (e.g. [12]). The third one is, precisely, Corollary 3.8 in [6]. erties for $Z^{t}$.

Using the properties of flows $X^{t}$ and $Y^{t}$ in Lemma 2.1 and Lemma 2.3 we obtain similar prop-

Lemma 2.4. For any $r \in\{1,2\}$ and $u_{0} \in H^{r}(\mathbb{R})$, the flow $Z^{t}$ satisfies the following estimate

$$
\left\|Z^{t} u_{0}\right\|_{H^{r}(\mathbb{R})} \leq C\left(\left\|u_{0}\right\|_{L^{2}(\mathbb{R})}\right)\left\|u_{0}\right\|_{H^{r}(\mathbb{R})}, \quad \forall t \in[0,1] .
$$

Moreover,

$$
\left\|Z^{t} u_{0}\right\|_{L^{2}(\mathbb{R})} \leq\left\|u_{0}\right\|_{L^{2}(\mathbb{R})}, \quad \forall t \geq 0 .
$$


2.2. The nonlinear flow $S^{t}$. Let us now focus on the nonlinear flow $S^{t}$ given by (1.1). Before entering in technical results, let us say a few words about the well-posedness of system (1.1). In [16] we proved that, for any initial data $u_{0} \in L^{1}(\mathbb{R})$, there exists a unique global solution $u \in C\left([0, \infty), L^{1}(\mathbb{R})\right)$. Moreover, for any $1<p<\infty$, it satisfies the following regularity:

$$
u \in C\left((0, \infty), W^{2, p}(\mathbb{R})\right) \cap C^{1}\left((0, \infty), L^{p}(\mathbb{R})\right) .
$$

Regarding the well-posedness in $H^{n}(\mathbb{R}), n \geq 1$, for any $u_{0} \in H^{n}(\mathbb{R})$ there exists a unique global solution $u \in C\left([0, \infty), H^{n}(\mathbb{R})\right)$. This can be proved by classical standard arguments for nonlinear evolution equations (see for example [8, Ch. 4]) and the variation of constants formula

$$
u(t)=G_{t} * X^{t} u_{0}+\frac{1}{2} \int_{0}^{t} G_{t-\tau} * X^{t-\tau}\left(u^{2}\right)_{x}(\tau) d \tau,
$$

where $G_{t}$ denotes the heat kernel.

In the following, we first obtain estimates on the $H^{n}(\mathbb{R})$-norm, $n \geq 0$ being an integer. We also study the $L^{2}(\mathbb{R})$ Lipschitz property of the flow $S^{t}$ in balls of $H^{1}(\mathbb{R})$.

Lemma 2.5. Let $T>0$. For every $u_{0} \in H^{n}(\mathbb{R})$, with $n \in \mathbb{N}$, the solution $S^{t} u_{0}$ of (1.1) satisfies

$$
\left\|S^{t} u_{0}\right\|_{H^{n}(\mathbb{R})} \leq C\left(T,\left\|u_{0}\right\|_{H^{n-1}(\mathbb{R})}\right)\left\|u_{0}\right\|_{H^{n}(\mathbb{R})}, \quad \forall t \in[0, T] .
$$

Moreover,

$$
\left\|S^{t} u_{0}\right\|_{L^{2}(\mathbb{R})} \leq\left\|u_{0}\right\|_{L^{2}(\mathbb{R})}, \quad \forall t \in[0, T]
$$

Proof. The $L^{2}(\mathbb{R})$-stability of the flow $S^{t}$ has already been proved in [16]. Indeed, multiplying equation (1.1) by $u(t)$ and using integration by parts one can obtain that

$$
\left\|S_{t} u_{0}\right\|_{L^{2}(\mathbb{R})} \leq\left\|u_{0}\right\|_{L^{2}(\mathbb{R})} .
$$

We now recall some well-known results about the $L^{p}(\mathbb{R})$-norms of the heat kernel (just use the fact that $G_{1}$ is a Schwartz function and $G_{t}$ scales correspondingly):

$$
\left\|\partial_{x}^{k} G_{t}\right\|_{L^{p}(\mathbb{R})} \simeq t^{-\frac{1}{2}\left(1-\frac{1}{p}\right)-\frac{k}{2}}, \quad, \forall k \in \mathbb{N} \cup\{0\}, \forall p \in[1, \infty] .
$$

Let us now consider the case $n \geq 1$. We know that $S^{t} u_{0}=u(t)$ satisfies the variation of constants formula (2.3). Differentiating identity (2.3) in the space variable $x$ and using that $X^{t}$ commutes with $\partial_{x}$, we obtain

$$
u_{x}(t)=G_{t} * X^{t}\left(\partial_{x} u_{0}\right)+\frac{1}{2} \int_{0}^{t} \partial_{x} G_{t-\tau} * X^{t-\tau}\left(u u_{x}(\tau)\right) d \tau .
$$

Using Young's inequality, Lemma 2.1 and the estimates in (2.4), we have that

$$
\begin{aligned}
\left\|u_{x}(t)\right\|_{H^{n-1}(\mathbb{R})} \lesssim & \left\|G_{t}\right\|_{L^{1}(\mathbb{R})}\left\|X^{t}\left(\partial_{x} u_{0}\right)\right\|_{H^{n-1}(\mathbb{R})} \\
& \quad+\int_{0}^{t}\left\|\partial_{x} G_{t-\tau}\right\|_{L^{2}(\mathbb{R})}\left\|X^{t-\tau}\left(u u_{x}(\tau)\right)\right\|_{W^{n-1,1}(\mathbb{R})} d \tau \\
& \lesssim\left\|\partial_{x} u_{0}\right\|_{H^{n-1}(\mathbb{R})}+\int_{0}^{t}(t-\tau)^{-\frac{3}{4}}\left\|X^{t-\tau}\left(u u_{x}(\tau)\right)\right\|_{W^{n-1,1}(\mathbb{R})} d \tau \\
& \lesssim\left\|u_{0}\right\|_{H^{n}(\mathbb{R})}+\int_{0}^{t}(t-\tau)^{-\frac{3}{4}}\left\|u u_{x}(\tau)\right\|_{W^{n-1,1}(\mathbb{R})} d \tau .
\end{aligned}
$$


When $n=1$, Hölder's inequality and the $L^{2}(\mathbb{R})$-stability of the flow $S^{t}$ yield

$$
\begin{aligned}
\left\|u_{x}(t)\right\|_{L^{2}(\mathbb{R})} & \leq\left\|u_{0}\right\|_{H^{1}(\mathbb{R})}+C \int_{0}^{t}(t-\tau)^{-\frac{3}{4}}\|u(\tau)\|_{L^{2}(\mathbb{R})}\left\|u_{x}(\tau)\right\|_{L^{2}(\mathbb{R})} d \tau \\
& \leq\left\|u_{0}\right\|_{H^{1}(\mathbb{R})}+C\left\|u_{0}\right\|_{L^{2}(\mathbb{R})} \int_{0}^{t}(t-\tau)^{-\frac{3}{4}}\left\|u_{x}(\tau)\right\|_{L^{2}(\mathbb{R})} d \tau .
\end{aligned}
$$

Fractional Gronwall's Lemma [6, Lemma 2.4] gives us the desired estimate when $n=1$. The case $n>1$ follows by induction.

Lemma 2.6. Let $R, T>0$. There exists $\gamma=\gamma(R, T)<\infty$ such that, if

$$
\left\|u_{0}\right\|_{H^{1}(\mathbb{R})} \leq R \quad \text { and } \quad\left\|v_{0}\right\|_{H^{1}(\mathbb{R})} \leq R,
$$

then

$$
\left\|S^{t} u_{0}-S^{t} v_{0}\right\|_{L^{2}(\mathbb{R})} \leq \gamma\left\|u_{0}-v_{0}\right\|_{L^{2}(\mathbb{R})}, \quad \forall t \in[0, T] .
$$

Proof. Let $u$ and $v$ be the corresponding solutions of (1.1) with $u_{0}$ and $v_{0}$ initial data. Manipulating the variation of constants formula we obtain that

$$
(u-v)(t)=G_{t} * X^{t}\left(u_{0}-v_{0}\right)+\frac{1}{2} \int_{0}^{t} G_{t-s} * X^{t-s}\left(u^{2}-v^{2}\right)_{x}(s) d s .
$$

Using the $L^{1}(\mathbb{R})$-norm decay of $G_{t}$ and its first derivative, given by (2.4), we obtain

$$
\begin{aligned}
\|(u-v)(t)\|_{L^{2}(\mathbb{R}) \leq} & \left\|G_{t} * X^{t}\left(u_{0}-v_{0}\right)\right\|_{L^{2}(\mathbb{R})} \\
& +\frac{1}{2} \int_{0}^{t}\left\|\left(G_{t-s}\right)_{x} * X^{t-s}\left(u^{2}-v^{2}\right)(s)\right\|_{L^{2}(\mathbb{R})} d s \\
\lesssim & \left\|u_{0}-v_{0}\right\|_{L^{2}(\mathbb{R})}+\int_{0}^{t}(t-s)^{-\frac{1}{2}}\left\|\left(u^{2}-v^{2}\right)(s)\right\|_{L^{2}(\mathbb{R})} \\
\lesssim & \left\|u_{0}-v_{0}\right\|_{L^{2}(\mathbb{R})} \\
& +\int_{0}^{t}(t-s)^{-\frac{1}{2}}\|(u-v)(s)\|_{L^{2}(\mathbb{R})}\left(\|u(s)\|_{H^{1}(\mathbb{R})}+\|v(s)\|_{H^{1}(\mathbb{R})}\right) d s .
\end{aligned}
$$

By Lemma 2.5 there exists a constant $C=C\left(T,\left\|u_{0}\right\|_{L^{2}(\mathbb{R})},\left\|v_{0}\right\|_{L^{2}(\mathbb{R})}\right)$ such that

$$
\begin{aligned}
\|(u-v)(t)\|_{L^{2}(\mathbb{R})} \leq & \left\|u_{0}-v_{0}\right\|_{L^{2}(\mathbb{R})} \\
& +C\left(\left\|u_{0}\right\|_{H^{1}(\mathbb{R})}+\left\|v_{0}\right\|_{H^{1}(\mathbb{R})}\right) \int_{0}^{t}(t-s)^{-\frac{1}{2}}\|(u-v)(s)\|_{L^{2}(\mathbb{R})} d s .
\end{aligned}
$$

Finally, Fractional Gronwall's lemma yields the result.

2.3. Local error estimates. Before proving Theorem 1.1, we obtain the error estimates on $Z^{t}$ in the $L^{2}(\mathbb{R})$-norm. We state this result in the following proposition.

Proposition 2.1. Let $r \in\{1,2\}$ and $u_{0} \in H^{r}(\mathbb{R})$. Then, there exists a positive constant $C=C\left(\left\|u_{0}\right\|_{L^{2}(\mathbb{R})}\right)$ such that for every $t \in(0,1)$ the following holds

$$
\left\|S^{t} u_{0}-Z^{t} u_{0}\right\|_{L^{2}(\mathbb{R})} \leq C\left\|u_{0}\right\|_{H^{r}(\mathbb{R})}^{2} t^{1+\frac{r}{2}} .
$$

Proof. We write the variation of constants formula both for $S^{t} u_{0}=u(t)$ solution of (1.1) and $Z^{t}$ given by (1.5). We have that they satisfy

$$
u(t)=D_{t} * G_{t} * u_{0}+\frac{1}{2} \int_{0}^{t} D_{t-s} * G_{t-s} *\left(u^{2}(s)\right)_{x} d s
$$


and

$$
Z^{t} u_{0}=X^{t} Y^{t} u_{0}=D_{t} * Y^{t} u_{0}=D_{t} *\left(G_{t} * u_{0}+\frac{1}{2} \int_{0}^{t} G_{t-s} *\left(\left(Y^{s} u_{0}\right)^{2}\right)_{x} d s\right)
$$

Therefore,

$$
\begin{aligned}
\left\|u(t)-Z^{t} u_{0}\right\|_{L^{2}(\mathbb{R})} & \leq \frac{1}{2} \int_{0}^{t}\left\|D_{t-s} * G_{t-s} *\left(u^{2}(s)\right)_{x}-D_{t} * G_{t-s} *\left(\left(Y^{s} u_{0}\right)^{2}\right)_{x}\right\|_{L^{2}(\mathbb{R})} d s \\
& \leq \frac{1}{2} \int_{0}^{t}\left(\left\|R_{1}(s)\right\|_{L^{2}(\mathbb{R})}+\left\|R_{2}(s)\right\|_{L^{2}(\mathbb{R})}+\left\|R_{3}(s)\right\|_{L^{2}(\mathbb{R})}\right) d s
\end{aligned}
$$

where

$$
\begin{aligned}
& R_{1}(s)=D_{t-s} * G_{t-s} *\left(u^{2}(s)\right)_{x}-D_{t-s} * G_{t-s} *\left(\left(Z^{s} u_{0}\right)^{2}\right)_{x}, \\
& R_{2}(s)=D_{t-s} * G_{t-s} *\left(\left(Z^{s} u_{0}\right)^{2}\right)_{x}-D_{t} * G_{t-s} *\left(\left(Z^{s} u_{0}\right)^{2}\right)_{x}, \\
& R_{3}(s)=D_{t} * G_{t-s} *\left(\left(Z^{s} u_{0}\right)^{2}\right)_{x}-D_{t} * G_{t-s} *\left(\left(Y^{s} u_{0}\right)^{2}\right)_{x} .
\end{aligned}
$$

We now estimate each of these terms.

Estimate on $R_{1}$. Using Young's inequality and the $L^{2}(\mathbb{R})$-estimates on $\partial_{x} G_{t}, u(t)$ and $Z^{s} u_{0}$, we obtain

$$
\begin{aligned}
\left\|R_{1}(s)\right\|_{L^{2}(\mathbb{R})} & \leq\left\|D_{t-s}\right\|_{L^{1}(\mathbb{R})}\left\|\partial_{x} G_{t-s}\right\|_{L^{2}(\mathbb{R})}\left\|u^{2}(s)-\left(Z^{s} u_{0}\right)^{2}\right\|_{L^{1}(\mathbb{R})} \\
& \lesssim(t-s)^{-\frac{3}{4}}\left\|u(s)-Z^{s} u_{0}\right\|_{L^{2}(\mathbb{R})}\left(\|u(s)\|_{L^{2}(\mathbb{R})}+\left\|Z^{s} u_{0}\right\|_{L^{2}(\mathbb{R})}\right) \\
& \lesssim(t-s)^{-\frac{3}{4}}\left\|u_{0}\right\|_{L^{2}(\mathbb{R})}\left\|u(s)-Z^{s} u_{0}\right\|_{L^{2}(\mathbb{R})} .
\end{aligned}
$$

Estimate on $R_{2}$. For any $t \in(0,1)$, from Lemma 2.2 we have

$$
\begin{aligned}
\left\|R_{2}(s)\right\|_{L^{2}(\mathbb{R})} & =\left\|\left(D_{t-s}-D_{t}\right) * G_{t-s} *\left(\left(Z^{s} u_{0}\right)^{2}\right)_{x}\right\|_{L^{2}(\mathbb{R})} \lesssim\left\|\partial_{x}^{2-r} G_{t-s} * \partial_{x}^{r}\left(\left(Z^{s} u_{0}\right)^{2}\right)\right\|_{L^{2}(\mathbb{R})} \\
& \leq s\left\|\partial_{x}^{2-r} G_{t-s}\right\|_{L^{1}(\mathbb{R})}\left\|\partial_{x}^{r}\left(\left(Z^{s} u_{0}\right)^{2}\right)\right\|_{L^{2}(\mathbb{R})} \lesssim s(t-s)^{-\frac{2-r}{2}}\left\|\left(Z^{s} u_{0}\right)^{2}\right\|_{H^{r}(\mathbb{R})} .
\end{aligned}
$$

Since $H^{r}(\mathbb{R})$ is an algebra for $r \in\{1,2\}$, we obtain that

$$
\left\|R_{2}(s)\right\|_{L^{2}(\mathbb{R})} \lesssim s(t-s)^{-\frac{2-r}{2}}\left\|Z^{s} u_{0}\right\|_{H^{r}(\mathbb{R})}^{2} .
$$

Integrating on the time interval $[0, t]$ and using the estimates on the $H^{r}(\mathbb{R})$-norm of $X^{t}$ and $Y^{t}$ in Lemma 2.1 and Lemma 2.3, we obtain

$$
\begin{aligned}
\int_{0}^{t}\left\|R_{2}(s)\right\|_{L^{2}(\mathbb{R})} d s & \lesssim\left\|Z^{s} u_{0}\right\|_{L^{\infty}\left((0, t), H^{r}(\mathbb{R})\right)}^{2} \int_{0}^{t} s(t-s)^{-\frac{2-r}{2}} d s \\
& \leq C\left(\left\|u_{0}\right\|_{L^{2}(\mathbb{R})}\right)\left\|u_{0}\right\|_{H^{r}(\mathbb{R})}^{2} t^{1+\frac{r}{2}}
\end{aligned}
$$

Estimate on $R_{3}$. Observe that

$$
\begin{aligned}
\left\|R_{3}(s)\right\|_{L^{2}(\mathbb{R})} & =\left\|D_{t} * G_{t-s} *\left(\left(Z^{s} u_{0}\right)^{2}-\left(Y^{s} u_{0}\right)^{2}\right)_{x}\right\|_{L^{2}(\mathbb{R})} \\
& \leq\left\|G_{t-s} *\left(\left(Z^{s} u_{0}\right)^{2}-\left(Y^{s} u_{0}\right)^{2}\right)_{x}\right\|_{L^{2}(\mathbb{R})} .
\end{aligned}
$$


We distinguish between the cases $r=1$ and $r=2$. When $r=1$, using Young's inequality, Lemma 2.1, Lemma 2.3 and the embedding $H^{1}(\mathbb{R}) \hookrightarrow L^{\infty}(\mathbb{R})$, we obtain:

$$
\begin{aligned}
\left\|R_{3}(s)\right\|_{L^{2}(\mathbb{R})} & \leq\left\|\partial_{x} G_{t-s}\right\|_{L^{1}(\mathbb{R})}\left\|\left(Z^{s} u_{0}\right)^{2}-\left(Y^{s} u_{0}\right)^{2}\right\|_{L^{2}(\mathbb{R})} \\
& \lesssim(t-s)^{-\frac{1}{2}}\left\|X^{s} Y^{s} u_{0}-Y^{s} u_{0}\right\|_{L^{2}(\mathbb{R})}\left(\left\|Z^{s} u_{0}\right\|_{L^{\infty}(\mathbb{R})}+\left\|Y^{s} u_{0}\right\|_{L^{\infty}(\mathbb{R})}\right) \\
& \lesssim s(t-s)^{-\frac{1}{2}}\left\|Y^{s} u_{0}\right\|_{H^{1}(\mathbb{R})}^{2} .
\end{aligned}
$$

For $r=2$, we proceed as follows:

$$
\begin{aligned}
\left\|R_{3}(s)\right\|_{L^{2}(\mathbb{R})} & \left.\leq\left\|G_{t-s}\right\|_{L^{1}(\mathbb{R})} \|\left(Z^{s} u_{0}\right)^{2}-\left(Y^{s} u_{0}\right)^{2}\right) \|_{H^{1}(\mathbb{R})} \\
& \leq\left\|Z^{s} u_{0}-Y^{s} u_{0}\right\|_{H^{1}(\mathbb{R})}\left\|X^{s} Y^{s} u_{0}+Y^{s} u_{0}\right\|_{H^{1}(\mathbb{R})} \\
& \lesssim\left\|Z^{s} u_{0}-Y^{s} u_{0}\right\|_{H^{1}(\mathbb{R})}\left\|Y^{s} u_{0}\right\|_{H^{1}(\mathbb{R})} \lesssim s\left\|Y^{s} u_{0}\right\|_{H^{2}(\mathbb{R})}^{2} .
\end{aligned}
$$

Therefore, in both cases $r \in\{1,2\}$ we have

$$
\begin{aligned}
\int_{0}^{t}\left\|R_{3}(s)\right\|_{L^{2}(\mathbb{R})} & \lesssim\left\|Y^{s} u_{0}\right\|_{L^{\infty}\left((0, t), H^{r}(\mathbb{R})\right)}^{2} \int_{0}^{t}(t-s)^{\frac{r-2}{2}} s d s \\
& \leq C\left(\left\|u_{0}\right\|_{L^{2}(\mathbb{R})}\right)\left\|u_{0}\right\|_{H^{r}(\mathbb{R})}^{2} t^{1+\frac{r}{2}}
\end{aligned}
$$

Finally, plugging estimates (2.7), (2.8) and (2.9) into (2.6), we obtain

$$
\begin{aligned}
\left\|u(t)-Z^{t} u_{0}\right\|_{L^{2}(\mathbb{R})} \leq C\left(\left\|u_{0}\right\|_{L^{2}(\mathbb{R})}\right)\left\|u_{0}\right\|_{\left.H^{r}(\mathbb{R})\right)^{2}}^{2} t^{1+\frac{r}{2}} & \\
& +C\left(\left\|u_{0}\right\|_{L^{2}(\mathbb{R})}\right) \int_{0}^{t}(t-s)^{-\frac{3}{4}}\left\|u(s)-Z^{s} u_{0}\right\|_{L^{2}(\mathbb{R})} d s .
\end{aligned}
$$

Applying fractional Gronwall's Lemma [6, Lemma 2.5] we conclude that

$$
\left\|u(t)-Z^{t} u_{0}\right\|_{L^{2}(\mathbb{R})} \leq C\left(\left\|u_{0}\right\|_{L^{2}(\mathbb{R})}\right)\left\|u_{0}\right\|_{H^{r}(\mathbb{R})^{t}}^{2} t^{1+\frac{r}{2}} .
$$

The proof is now finished.

2.4. Proof of Theorem 1.1. We are now able to prove Theorem 1.1. It is enough to follow "Lady Windermere's fan" argument (as in [14]; see also [6]). Let us denote by $u^{n}:=Z^{n \Delta t} u_{0}$ the approximate solution and $u_{m}^{n}:=S^{(n-m) \Delta t} u^{m}$. Note that $u^{n}=u_{n}^{n}$ and $u(n \Delta t)=u_{0}^{n}$.

Let us first observe a few properties of the sequence $u^{n}$. Lemma 2.4 shows that

$$
\left\|u^{n}\right\|_{L^{2}(\mathbb{R})} \leq\left\|u_{0}\right\|_{L^{2}(\mathbb{R})}, \quad \forall n \geq 0 .
$$

Let us now consider the case of the $H^{r}(\mathbb{R})$ norms, $r \in\{1,2\}$. First, recall that by Lemma 2.3 and estimate (2.10) there exists a positive constant $C=C\left(\left\|u_{0}\right\|_{L^{2}(\mathbb{R})}\right)$ such that for any $\Delta t \leq 1$ the following holds:

This implies that

$$
\left\|Y^{\Delta t} u^{m}\right\|_{H^{r}(\mathbb{R})} \leq e^{C \Delta t}\left\|u^{m}\right\|_{H^{r}(\mathbb{R})}, \quad \forall m \geq 0 .
$$

$$
\left\|Z^{n \Delta t} u_{0}\right\|_{H^{r}(\mathbb{R})}=\left\|X^{\Delta t} Y^{\Delta t} u^{n-1}\right\|_{H^{r}(\mathbb{R})} \leq e^{C \Delta t}\left\|u^{n-1}\right\|_{H^{r}(\mathbb{R})} \leq e^{C n \Delta t}\left\|u_{0}\right\|_{H^{r}(\mathbb{R})}, \forall n \geq 0 .
$$

As long as $n \Delta t \leq T$, we get that $u^{n}=Z^{n \Delta t} u_{0}$ satisfies

$$
\left\|Z^{n \Delta t} u_{0}\right\|_{H^{r}(\mathbb{R})} \leq e^{C T}\left\|u_{0}\right\|_{H^{r}(\mathbb{R})}
$$

and, hence, the second part of Theorem 1.1 is proved. 
We now proceed to obtain the global error estimate. We have

$$
\begin{aligned}
\left\|Z^{n \Delta t} u_{0}-u(n \Delta t)\right\|_{L^{2}(\mathbb{R})} & \leq \sum_{m=0}^{n-1}\left\|u_{m+1}^{n}-u_{m}^{n}\right\|_{L^{2}(\mathbb{R})} \\
& =\sum_{m=0}^{n-1}\left\|S^{(n-m-1) \Delta t}\left(Z^{\Delta t} u^{m}\right)-S^{(n-m-1) \Delta t}\left(S^{\Delta t} u^{m}\right)\right\|_{L^{2}(\mathbb{R})}
\end{aligned}
$$

In order to apply Lemma 2.6, we need to guarantee that $Z^{\Delta t} u^{m}=Z^{(m+1) \Delta t} u_{0}$ and $S^{\Delta t} u^{m}$ belong to some fix ball of $H^{1}(\mathbb{R})$ for $0 \leq m \leq n-1$. For $Z^{\Delta t} u^{m}$ we proved such an estimate in (2.11). For the second term, since $\Delta t \leq 1$, by Lemma 2.5 we have

$$
\left\|S^{\Delta t} u^{m}\right\|_{H^{1}(\mathbb{R})} \leq C\left(1,\left\|u_{0}\right\|_{L^{2}(\mathbb{R})}\right)\left\|u^{m}\right\|_{H^{1}(\mathbb{R})} \leq C\left(\left\|u_{0}\right\|_{L^{2}(\mathbb{R})}\right) e^{T C\left(\left\|u_{0}\right\|_{L^{2}(\mathbb{R})}\right)}\left\|u_{0}\right\|_{H^{1}(\mathbb{R})} .
$$

We now apply Lemma 2.6 with $r=1$. This gives us that, for some constant $C=C\left(T,\left\|u_{0}\right\|_{H^{1}(\mathbb{R})}\right)$,

$$
\left\|Z^{n \Delta t} u_{0}-u(n \Delta t)\right\|_{L^{2}(\mathbb{R})} \leq C \sum_{m=0}^{n-1}\left\|Z^{\Delta t} u^{m}-S^{\Delta t} u^{m}\right\|_{L^{2}(\mathbb{R})} .
$$

Using the local error estimate obtained in Proposition 2.1, we finally conclude that

$$
\left\|Z^{n \Delta t} u_{0}-u(n \Delta t)\right\|_{L^{2}(\mathbb{R})} \leq C \sum_{m=0}^{n-1}\left\|u^{m}\right\|_{H^{r}(\mathbb{R})}^{2}(\Delta t)^{1+\frac{r}{2}} \leq C\left(T,\left\|u_{0}\right\|_{H^{1}}\right)\left\|u_{0}\right\|_{H^{r}(\mathbb{R})}^{2}(\Delta t)^{\frac{r}{2}} .
$$

The proof of the global error estimate is complete.

\section{LARGE-TIME BEHAVIOR}

In this section, we first analyze the decay properties of $u^{\Delta}$ and then we prove Theorem 1.2. We extend the splitting (1.5) to a continuous-in-time single equation in order to use a scaling argument, as in [16]. Hence, we first observe that $u^{\Delta}$, defined by (1.6), satisfies the following equation:

$$
\left\{\begin{array}{l}
u_{t}^{\Delta}=2 \psi^{\Delta t}(t) u_{x x}^{\Delta}+\psi^{\Delta t}(t)\left(\left(u^{\Delta}\right)^{2}\right)_{x} \\
\quad+2\left(1-\psi^{\Delta t}(t)\right)\left(K * u^{\Delta}-u^{\Delta}+u_{x}^{\Delta}\right), \quad \forall t \in(0, \infty) \backslash \Delta, \\
u^{\Delta}(0)=u_{0},
\end{array}\right.
$$

where $\psi^{\Delta t}(t):=\sum_{n \geq 0} \chi_{I_{n}^{\Delta}}(t)$ and $I_{n}^{\Delta}:=\left(t_{n}, t_{n+1 / 2}\right)$. Here,

$$
\chi_{S}(t)= \begin{cases}1, & t \in S \\ 0, & t \notin S\end{cases}
$$

denotes the characteristic function of a given set $S \subset \mathbb{R}$. We also emphasize that the regularity of $u^{\Delta}$ in (1.7) shows that the time derivative in the first equation of system (3.1) may be also understood in the weak sense.

Let us now explain the main steps of this section, whose main objective is the proof of Theorem 1.2. We first obtain estimates on the $L^{p}(\mathbb{R})$-norms, $1 \leq p<\infty$, of $u^{\Delta}$. This is done by adapting the methods developed in [18] to system (3.1). Next, in Section 3.2 we introduce a family of rescaled solutions $\left\{u_{\lambda}\right\}_{\lambda>0}$ and obtain uniform, with respect to $\lambda$, estimates that will allow us to prove the compactness of the family. Finally, we prove Theorem 1.2 in Section 3.4. The main idea is that the desired asymptotic expansion on (1.8) is equivalent to the fact that, for some positive time $t_{0}$, 
$u^{\lambda}\left(t_{0}\right) \rightarrow u_{M}\left(t_{0}\right)$ in the $L^{1}(\mathbb{R})$-norm. To prove that, we use the compactness of $\left\{u_{\lambda}\right\}_{\lambda>0}$ in Section 3.3 and show that any accumulation point verifies equation (1.9).

3.1. Estimates for the $L^{p}(\mathbb{R})$-norm. First, we need to obtain decay estimates for the $L^{p}$-norms of $u^{\Delta}$. We proceed as in $[18,19]$.

Lemma 3.1. For any $u_{0} \in L^{1}(\mathbb{R}) \cap L^{\infty}(\mathbb{R})$ and $p \in[1, \infty)$ there exists a positive constant

$$
C=C\left(p,\left\|u_{0}\right\|_{L^{1}(\mathbb{R})},\left\|u_{0}\right\|_{L^{\infty}(\mathbb{R})}\right)>0
$$

such that the solution of system (3.1) satisfies

$$
\left\|u^{\Delta}(t)\right\|_{L^{p}(\mathbb{R})} \leq C(t+1)^{-\frac{1}{2}\left(1-\frac{1}{p}\right)}, \quad \forall t>0 .
$$

Moreover, the $L^{1}(\mathbb{R})$-norm of $u^{\Delta}$ does not increase.

Proof. For $p=1$ the desired estimate on $u^{\Delta}$ follows from the definition of (1.6) and the $L^{1}(\mathbb{R})$ stability property of the flows $X^{t}$ and $Y^{t}$. Let us now consider $p=2^{k}$ where $k \geq 1$ is an integer. We emphasize that, in order to prove the decay property (3.2), it is sufficient to consider these particular cases of $p$. As a matter of fact, all the computations done below are well defined due to that particular choice. To simplify the presentation we will denote by $u$ the solution of equation (3.1). Multiplying equation (3.1) by $u^{p-1}$ and integrating on $\mathbb{R}$, we obtain

$$
\begin{aligned}
\frac{1}{p} \frac{d}{d t}\|u(t)\|_{L^{p}(\mathbb{R})}^{p}=- & C(p) \psi^{\Delta t}(t)\left\|\left(u^{p / 2}\right)_{x}(t)\right\|_{L^{2}(\mathbb{R})}^{2} \\
& +2\left(1-\psi^{\Delta t}(t)\right) \int_{\mathbb{R}}(K * u-u) u^{p-1}(t, x) d x \\
= & I_{1}+I_{2} .
\end{aligned}
$$

Let us denote $J(z)=\frac{1}{2}(K(z)+K(-z))$. We have that $J$ is a positive even function with mass one having a second momentum in $L^{1}(\mathbb{R})$. In our particular case its Fourier transform is given by

$$
\widehat{J}(\xi)=\frac{1}{1+\xi^{2}} .
$$

The above properties imply that $J$ satisfies the hypotheses in [18, Lemma 5.1], i.e. there exist positive constants $A, R$ and $\delta$ such that $\widehat{J}(\xi) \leq 1-A \xi^{2}$ for $|\xi| \leq R$ and $\widehat{J}(\xi) \leq 1-\delta$ for any $|\xi|>R$. This will be essential in applying the method in [18] to obtain the decay of the $L^{p}(\mathbb{R})$-norms of the solutions.

Using the inequality (see [15, Lemma 2.3] for full details)

$$
\int_{\mathbb{R}} \int_{\mathbb{R}} J(x-y)(\varphi(x)-\varphi(y))^{2} d x d y \leq \int_{\mathbb{R}} J(z) z^{2} d z \int_{\mathbb{R}} \varphi_{x}^{2} d x=2\left\|\varphi_{x}\right\|_{L^{2}(\mathbb{R})}^{2},
$$

with $\varphi=u^{p / 2}$ we obtain that the first term in the right hand side of (3.3) satisfies

$$
I_{1} \leq-C(p, J) \psi^{\Delta t}(t) \int_{\mathbb{R}} \int_{\mathbb{R}} J(x-y)\left(u^{p / 2}(x)-u^{p / 2}(y)\right)^{2} d x d y .
$$


We now prove a similar estimate for $I_{2}$. Elementary inequalities, such as Young's inequality, and the fact that $K$ has mass one show that the following holds:

$$
\begin{aligned}
\iint_{\mathbb{R}^{2}} K(x-y) u(y) u^{p-1}(x) d x d y \leq & \frac{2}{p} \iint_{\mathbb{R}^{2}} K(x-y) u^{p / 2}(y) u^{p / 2}(x) d x d y \\
& +\left(1-\frac{2}{p}\right) \int_{\mathbb{R}} u^{p}(x) d x
\end{aligned}
$$

This implies that $I_{2}$ satisfies

$$
I_{2} \leq \frac{4}{p}\left(1-\psi^{\Delta t}(t)\right) \int_{\mathbb{R}}\left(K * u^{p / 2}-u^{p / 2}\right) u^{p / 2} .
$$

Note that for any function $\varphi \in L^{2}(\mathbb{R})$ we have

$$
\begin{aligned}
\int_{\mathbb{R}}(K * \varphi-\varphi) \varphi d x & =-\frac{1}{2} \int_{\mathbb{R}} \int_{\mathbb{R}} K(x-y)(\varphi(x)-\varphi(y))^{2} d x d y \\
& =-\frac{1}{2} \int_{\mathbb{R}} \int_{\mathbb{R}} J(x-y)(\varphi(x)-\varphi(y))^{2} d x d y .
\end{aligned}
$$

Therefore, we have that $I_{2}$ satisfies

$$
I_{2} \leq-\frac{2}{p}\left(1-\psi^{\Delta t}(t)\right) \int_{\mathbb{R}} \int_{\mathbb{R}} J(x-y)\left(u^{p / 2}(x)-u^{p / 2}(y)\right)^{2} d x d y .
$$

Using estimates (3.5) and (3.7) in (3.3) we obtain that, for some positive constant $C(J, p)$ we have the following differential inequality for any $t \in(0, \infty) \backslash \Delta$ :

$$
\frac{d}{d t}\|u(t)\|_{L^{p}(\mathbb{R})}^{p} \leq-C(J, p) \int_{\mathbb{R}} \int_{\mathbb{R}} J(x-y)\left(u^{p / 2}(t, x)-u^{p / 2}(t, y)\right)^{2} d x d y .
$$

When $p=2$, we use the arguments in [18, Lemma 5.1] on each of the intervals $\left(t_{n}, t_{n+1 / 2}\right)$ and $\left(t_{n+1 / 2}, t_{n+1}\right), n \geq 0$. We obtain the existence of a positive constant $a$, depending on $J$ but not on $\Delta t$, such that the following holds:

$$
\frac{d}{d t}\left[(t+a)\|u(t)\|_{L^{2}(\mathbb{R})}^{2}\right] \lesssim(t+a)^{-1 / 2}\left\|u_{0}\right\|_{L^{1}(\mathbb{R})}^{2} \quad \forall t \in(0, \infty) \backslash \Delta .
$$

Integrating the above inequality on the intervals $\left(t_{n}, t_{n+1 / 2}\right)$ and $\left(t_{n+1 / 2}, t_{n+1}\right), n \geq 0$, summing up the resulted inequalities and rescaling parameter $a$ we conclude that

$$
\left\|u^{\Delta}(t)\right\|_{L^{2}(\mathbb{R})} \lesssim \frac{\left\|u_{0}\right\|_{L^{2}(\mathbb{R})}}{(t+1)^{1 / 2}}+\frac{\left\|u_{0}\right\|_{L^{1}(\mathbb{R})}}{(t+1)^{1 / 4}}
$$

Using estimate (3.8) and proceeding as in the proof of [18, Theorem 1.4], we obtain the estimates for the remaining $L^{p}(\mathbb{R})$-norms for $p>2$.

3.2. Estimates on the rescaled solutions. In order to prove Theorem 1.2, we introduce the following family of rescaled functions. Given $\lambda>0$, we define $u^{\lambda}$ by

$$
u^{\lambda}(t, x)=\lambda u^{\Delta}\left(\lambda^{2} t, \lambda x\right)
$$


It follows that $u^{\lambda}$ is the solution of the following rescaled system:

$$
\left\{\begin{array}{l}
u_{t}^{\lambda}=2 \psi^{\frac{\Delta t}{\lambda^{2}}}(t) u_{x x}^{\lambda}+2 \psi^{\frac{\Delta t}{\lambda^{2}}}(t) u^{\lambda} u_{x}^{\lambda} \\
\quad+2\left(1-\psi^{\frac{\Delta t}{\lambda^{2}}}(t)\right)\left(\lambda^{2} K_{\lambda} * u^{\lambda}-\lambda^{2} u^{\lambda}+\lambda u_{x}^{\lambda}\right), \quad \forall t \in(0, \infty) \backslash \lambda^{-2} \Delta, \\
u^{\lambda}(0, x)=\lambda u_{0}(\lambda x),
\end{array}\right.
$$

where $K_{\lambda}(z)=\lambda K(\lambda z)$. Note that we used

$$
\psi^{\Delta t}\left(\lambda^{2} t\right)=\sum_{n \geq 1} \chi_{\left(n \Delta t<\lambda^{2} t<\left(n+\frac{1}{2}\right) \Delta t\right)}=\sum_{n \geq 1} \chi_{\left(\frac{n \Delta t}{\lambda^{2}}<t<\left(n+\frac{1}{2}\right) \frac{\Delta t}{\lambda^{2}}\right)}=\psi^{\frac{\Delta t}{\lambda^{2}}}(t)
$$

in order to obtain the equation satisfied by $u^{\lambda}$.

The results in Lemma 3.1 extend immediately to the family $\left\{u^{\lambda}\right\}_{\lambda>0}$.

Lemma 3.2. Let $u_{0} \in L^{1}(\mathbb{R}) \cap L^{\infty}(\mathbb{R})$. For any $p \in[1, \infty)$ there exists a positive constant $C=$ $C\left(p,\left\|u_{0}\right\|_{L^{1}(\mathbb{R})},\left\|u_{0}\right\|_{L^{\infty}(\mathbb{R})}\right)$ such that

$$
\left\|u^{\lambda}(t)\right\|_{L^{p}(\mathbb{R})} \leq C t^{-\frac{1}{2}\left(1-\frac{1}{p}\right)},
$$

holds for any $\lambda>0$. Moreover, the mass of $u^{\lambda}$ is conserved.

Proof. By the definition of $u^{\lambda}$ and Lemma 3.1, we have

$$
\left\|u^{\lambda}(t)\right\|_{L^{p}(\mathbb{R})}=\lambda^{1-\frac{1}{p}}\left\|u^{\Delta}\left(\lambda^{2} t\right)\right\|_{L^{p}(\mathbb{R})} \leq C\left(t+\lambda^{-2}\right)^{-\frac{1}{2}\left(1-\frac{1}{p}\right)} \leq C t^{-\frac{1}{2}\left(1-\frac{1}{p}\right)} .
$$

The proof is now finished. The mass conservation follows from the same property of $X^{t}$ and $Y^{t}$. argument:

In order to prove the compactness of the family $\left\{u^{\lambda}\right\}_{\lambda>0}$, we recall the following compactness

Theorem 3.1. ([15, Theorem 3.1]) Let $1 \leq p<\infty$ and $\Omega \subset \mathbb{R}^{d}$ be an open set. Let $\rho: \mathbb{R}^{d} \rightarrow \mathbb{R}$ be a nonnegative smooth radial function with compact support, not identically zero, and $\rho_{n}(x)=n^{d} \rho(n x)$. Let $\left\{f_{n}\right\}_{n \geq 1}$ be a sequence of functions in $L^{p}((0, T) \times \Omega)$ such that

$$
\int_{0}^{T} \int_{\Omega}\left|f_{n}(t, x)\right|^{p} d x d t \leq M
$$

and

$$
n^{p} \int_{0}^{T} \int_{\Omega} \int_{\Omega} \rho_{n}(x-y)\left|f_{n}(t, x)-f_{n}(t, y)\right|^{p} d x d y d t \leq M
$$

Then, we have:

(1) If $\left\{f_{n}\right\}_{n \geq 1}$ is weakly convergent in $L^{p}((0, T) \times \Omega)$ to $f$, then $f \in L^{p}\left((0, T), W^{1, p}(\Omega)\right)$ for $p>1$ and $f \in L^{1}((0, T), B V(\Omega))$ for $p=1$.

(2) Let $p>1$. Assuming that $\Omega$ is a smooth bounded domain in $\mathbb{R}^{d}, \rho(x) \geq \rho(y)$ if $|x| \leq|y|$ and that

$$
\left\|\partial_{t} f_{n}\right\|_{L^{p}\left((0, T), W^{-1, p}(\Omega)\right)} \leq M
$$

then $\left\{f_{n}\right\}_{n \geq 1}$ is relatively compact in $L^{p}((0, T) \times \Omega)$. 
We will apply the above theorem with $p=2$ to obtain the local compactness in $L_{l o c}^{2}((0, \infty) \times \mathbb{R})$. Let us consider $0<\tau_{1}<\tau_{2}<\infty$. The boundedness of $\left\{u_{\lambda}\right\}_{\lambda>0}$ in $L^{2}\left(\left(\tau_{1}, \tau_{2}\right) \times \mathbb{R}\right)$ follows from Lemma 3.2. We now check the other assumptions in Theorem 3.1. In what follows we denote $J_{\lambda}(z)=$ $\lambda J(\lambda z)$.

Lemma 3.3. There exists a positive constant $C=C\left(J,\left\|u_{0}\right\|_{L^{1}(\mathbb{R})},\left\|u_{0}\right\|_{L^{\infty}(\mathbb{R})}\right)$ such that for any $0<$ $\tau_{1}<\tau_{2}<\infty$, the estimate

$$
\lambda^{2} \int_{\tau_{1}}^{\tau_{2}} \int_{\mathbb{R}} \int_{\mathbb{R}} J_{\lambda}(x-y)\left(u^{\lambda}(t, x)-u^{\lambda}(t, y)\right)^{2} d x d y d t \leq C \tau_{1}^{-\frac{1}{2}}
$$

holds for all $\lambda>0$.

Proof. Let us multiply (3.10) by $u^{\lambda}$ and integrate it on space. Using (3.6) for any $t \in(0, \infty) \backslash \lambda^{-2} \Delta$ we get the following identity:

$$
\begin{aligned}
\frac{1}{4} \frac{d}{d t} \int_{\mathbb{R}}\left|u^{\lambda}\right|^{2} d x= & -\psi^{\frac{\Delta t}{\lambda^{2}}}(t) \int_{\mathbb{R}}\left|u_{x}^{\lambda}\right|^{2} d x+\lambda^{2}\left(1-\psi^{\frac{\Delta t}{\lambda^{2}}}(t)\right) \int_{\mathbb{R}}\left(K_{\lambda} * u^{\lambda}-u^{\lambda}\right) u^{\lambda} d x \\
= & -\psi^{\frac{\Delta t}{\lambda^{2}}}(t) \int_{\mathbb{R}}\left|u_{x}^{\lambda}\right|^{2} d x \\
& \quad-\frac{\lambda^{2}}{2}\left(1-\psi^{\frac{\Delta t}{\lambda^{2}}}(t)\right) \int_{\mathbb{R}} \int_{\mathbb{R}} J_{\lambda}(x-y)\left(u^{\lambda}(x)-u^{\lambda}(y)\right)^{2} d x d y .
\end{aligned}
$$

Integrating on $\left(\tau_{1}, \tau_{2}\right)$ and using the decay of the $L^{2}(\mathbb{R})$-norm of $u^{\lambda}$ we obtain

$$
\begin{aligned}
& \int_{\tau_{1}}^{\tau_{2}} \psi^{\frac{\Delta t}{\lambda^{2}}}(t) \int_{\mathbb{R}}\left|u_{x}^{\lambda}\right|^{2} d x d t \\
& \quad+\lambda^{2} \int_{\tau_{1}}^{\tau_{2}}\left(1-\psi^{\frac{\Delta t}{\lambda^{2}}}(t)\right) \int_{\mathbb{R}} \int_{\mathbb{R}} J_{\lambda}(x-y)\left(u^{\lambda}(x)-u^{\lambda}(y)\right)^{2} d x d y d t \\
& \lesssim\left\|u^{\lambda}\left(\tau_{1}\right)\right\|_{L^{2}(\mathbb{R})}^{2}-\left\|u^{\lambda}\left(\tau_{2}\right)\right\|_{L^{2}(\mathbb{R})}^{2} \leq C\left(\left\|u_{0}\right\|_{L^{1}(\mathbb{R})},\left\|u_{0}\right\|_{L^{\infty}(\mathbb{R})}\right) \tau_{1}^{-\frac{1}{2}}
\end{aligned}
$$

By (3.4) and a change of variables, we also have

$$
\lambda^{2} \int_{\mathbb{R}} \int_{\mathbb{R}} J_{\lambda}(x-y)\left(u^{\lambda}(x)-u^{\lambda}(y)\right)^{2} d x d y \leq C(J) \int_{\mathbb{R}}\left|u_{x}^{\lambda}(x)\right|^{2} d x
$$

We introduce this inequality in (3.11) to obtain the desired result.

We now prove that the family $\left\{u_{\lambda}\right\}_{\lambda>0}$ satisfies the last assumption in Theorem 3.1.

Lemma 3.4. There exists a positive constant $C=C\left(J,\left\|u_{0}\right\|_{L^{1}(\mathbb{R})},\left\|u_{0}\right\|_{L^{\infty}(\mathbb{R})}\right)$ such that for any $0<$ $\tau_{1}<\tau_{2}<\infty$ the estimate

holds uniformly for all $\lambda>1$.

$$
\left\|u_{t}^{\lambda}\right\|_{L^{2}\left(\left(\tau_{1}, \tau_{2}\right), H^{-1}(\mathbb{R})\right)} \leq C \tau_{1}^{-\frac{1}{2}}
$$

Proof. Let us consider $\phi \in C_{c}^{\infty}(\mathbb{R})$ and $t \in\left(\tau_{1}, \tau_{2}\right) \backslash \lambda^{-2} \Delta$. Then

$$
\begin{gathered}
\left\langle u_{t}^{\lambda}(t), \phi\right\rangle_{-1,1}=\left\langle 2 \psi^{\frac{\Delta t}{\lambda^{2}}}(t) u_{x x}^{\lambda}+2 \psi^{\frac{\Delta t}{\lambda^{2}}}(t) u^{\lambda} u_{x}^{\lambda}+2\left(1-\psi^{\frac{\Delta t}{\lambda^{2}}}(t)\right)\left(\lambda^{2}\left(K_{\lambda} * u^{\lambda}-u^{\lambda}\right)+\lambda u_{x}^{\lambda}\right), \phi\right\rangle_{-1,1} \\
=-2 \psi^{\frac{\Delta t}{\lambda^{2}}}(t) \int_{\mathbb{R}} u_{x}^{\lambda} \phi_{x} d x-\psi^{\frac{\Delta t}{\lambda^{2}}}(t) \int_{\mathbb{R}}\left(u^{\lambda}\right)^{2} \phi_{x} d x \\
+2\left(1-\psi^{\frac{\Delta t}{\lambda^{2}}}(t)\right) \int_{\mathbb{R}}\left(\lambda^{2}\left(K_{\lambda} * u^{\lambda}-u^{\lambda}\right)+\lambda u_{x}^{\lambda}\right) \phi d x .
\end{gathered}
$$


Hence, for a.e. $t>0$ we have

$$
\begin{aligned}
\left|\left\langle u_{t}^{\lambda}(t), \phi\right\rangle_{-1,1}\right| \lesssim & \psi^{\frac{\Delta t}{\lambda^{2}}}(t)\left\|u_{x}^{\lambda}(t)\right\|_{L^{2}(\mathbb{R})}\|\phi\|_{H^{1}(\mathbb{R})}+\psi^{\frac{\Delta t}{\lambda^{2}}}(t)\left\|\left(u^{\lambda}\right)^{2}(t)\right\|_{L^{2}(\mathbb{R})}\|\phi\|_{H^{1}(\mathbb{R})} \\
& +\left(1-\psi^{\frac{\Delta t}{\lambda^{2}}}(t)\right)\left|\int_{\mathbb{R}}\left(\lambda^{2}\left(K_{\lambda} * u^{\lambda}-u^{\lambda}\right)+\lambda u_{x}^{\lambda}\right) \phi d x\right|
\end{aligned}
$$

Let us now estimate the last term in the right hand side of the above inequality. Let us first recall that the Fourier transforms of $K$ and $J$ are given by

$$
\widehat{K}(\xi)=\frac{1}{1+i \xi} \quad \text { and } \quad \widehat{J}(\xi)=\frac{1}{1+\xi^{2}} .
$$

The rescaled functions $K_{\lambda}$ and $J_{\lambda}$ satisfy

$$
\widehat{K}_{\lambda}(\xi)=\frac{1}{1+i \xi / \lambda} \quad \text { and } \quad \widehat{J}_{\lambda}(\xi)=\frac{1}{1+(\xi / \lambda)^{2}} .
$$

Thus

$$
\begin{aligned}
& \left|\int_{\mathbb{R}}\left(\lambda^{2}\left(K_{\lambda} * u^{\lambda}-u^{\lambda}\right)+\lambda u_{x}^{\lambda}\right) \phi d x\right| \leq \int_{\mathbb{R}}\left|\left(\lambda^{2}\left(\widehat{K}_{\lambda}(\xi)-1\right)+\lambda i \xi\right) \widehat{u^{\lambda}}(\xi) \widehat{\widehat{\phi}(\xi)} d \xi\right| \\
& \quad \leq \int_{\mathbb{R}} \frac{\lambda \xi^{2}}{\sqrt{\lambda^{2}+\xi^{2}}}\left|\widehat{u^{\lambda}}(\xi)\right||\widehat{\phi}(\xi)| d \xi \leq\left(\int_{\mathbb{R}} \frac{\lambda^{2} \xi^{2}}{\lambda^{2}+\xi^{2}}\left|\widehat{u^{\lambda}}(\xi)\right|^{2} d \xi\right)^{1 / 2}\|\phi\|_{H^{1}(\mathbb{R})} \\
& \quad=\left(\left.\int_{\mathbb{R}}\left(1-\widehat{J_{\lambda}}(\xi)\right) \widehat{u^{\lambda}}(\xi)\right|^{2} d \xi\right)^{1 / 2}\|\phi\|_{H^{1}(\mathbb{R})} \\
& \quad=\left(\frac{\lambda^{2}}{2} \int_{\mathbb{R}} J_{\lambda}(x-y)\left(u^{\lambda}(x)-u^{\lambda}(y)\right)^{2} d x d y\right)^{1 / 2}\|\phi\|_{H^{1}(\mathbb{R})} .
\end{aligned}
$$

Hence

$$
\begin{aligned}
\left|\left\langle u_{t}^{\lambda}(t), \phi\right\rangle_{-1,1}\right| & \lesssim \psi^{\frac{\Delta t}{\lambda^{2}}}(t)\|\phi\|_{H^{1}(\mathbb{R})}\left(\left\|u_{x}^{\lambda}(t)\right\|_{L^{2}(\mathbb{R})}+\left\|u^{\lambda}(t)\right\|_{L^{4}(\mathbb{R})}^{2}\right) \\
& +\left(1-\psi^{\frac{\Delta t}{\lambda^{2}}}(t)\right)\left(\frac{\lambda^{2}}{2} \int_{\mathbb{R}} J_{\lambda}(x-y)\left(u^{\lambda}(x)-u^{\lambda}(y)\right)^{2} d x d y\right)^{1 / 2}\|\phi\|_{H^{1}(\mathbb{R})} .
\end{aligned}
$$

Using the $L^{4}(\mathbb{R})$-decay of $u_{\lambda}$ in Lemma 3.2 we have

$$
\begin{aligned}
\left\|u_{t}^{\lambda}(t)\right\|_{H^{-1}(\mathbb{R})} \lesssim & \psi^{\frac{\Delta t}{\lambda^{2}}}(t)\left(\left\|u_{x}^{\lambda}(t)\right\|_{L^{2}(\mathbb{R})}+t^{-3 / 4}\right) \\
& +\left(1-\psi^{\frac{\Delta t}{\lambda^{2}}}(t)\right)\left(\frac{\lambda^{2}}{2} \int_{\mathbb{R}} J_{\lambda}(x-y)\left(u^{\lambda}(x)-u^{\lambda}(y)\right)^{2} d x d y\right)^{1 / 2}
\end{aligned}
$$

Integrating on time the above inequality, we get

$$
\begin{aligned}
\int_{\tau_{1}}^{\tau_{2}}\left\|u_{t}^{\lambda}(t)\right\|_{H^{-1}(\mathbb{R})}^{2} d t \lesssim & \tau_{1}^{-\frac{1}{2}}+\int_{\tau_{1}}^{\tau_{2}} \psi^{\frac{\Delta t}{\lambda^{2}}}(t) \int_{\mathbb{R}}\left|u_{x}^{\lambda}(t)\right|^{2} d t \\
& +\frac{\lambda^{2}}{2} \int_{\tau_{1}}^{\tau_{2}}\left(1-\psi^{\frac{\Delta t}{\lambda^{2}}}(t)\right) \int_{\mathbb{R}} J_{\lambda}(x-y)\left(u^{\lambda}(x)-u^{\lambda}(y)\right)^{2} d x d y d t
\end{aligned}
$$

Using now estimate (3.11) we conclude that

$$
\int_{\tau_{1}}^{\tau_{2}}\left\|u_{t}^{\lambda}(t)\right\|_{H^{-1}(\mathbb{R})}^{2} d t \lesssim \tau_{1}^{-\frac{1}{2}},
$$

which finishes the proof. 
The previous estimates obtained in Lemma 3.2, Lemma 3.3, Lemma 3.4 and Theorem 3.1 show that the family $\left\{u_{\lambda}\right\}_{\lambda>0}$ is relatively compact in $L_{l o c}^{2}((0, \infty) \times \mathbb{R})$ and so in $L_{l o c}^{1}((0, \infty) \times \mathbb{R})$. In order to prove the compactness in $L_{l o c}^{1}\left((0, \infty), L^{1}(\mathbb{R})\right)$ we need to uniformly control the tails. Before proving that, we need the following comparison result.

Lemma 3.5. Let $u_{0} \leq v_{0}$ be two functions in $L^{1}(\mathbb{R}) \cap L^{\infty}(\mathbb{R})$. Then, the solutions $u^{\lambda}$ and $v^{\lambda}$ of system (3.10) with initial data $u_{0}$ and $v_{0}$, respectively, satisfy

$$
u^{\lambda}(t, x) \leq v^{\lambda}(t, x)
$$

Proof. Using the definition of $u^{\lambda}$ and $v^{\lambda}$ it is sufficient to prove that $u^{\Delta}(t) \leq v^{\Delta}(t)$ holds for all $t>0$. In view of definition (1.6) and the comparison principle for $X^{t}$ and $Y^{t}$ given by Lemma 2.1 and Lemma 2.3 the property follows.

To conclude this subsection, let us show that the tails are uniformly small when $\lambda \geq 1$, a sufficient condition to prove, afterwards, that $\left\{u^{\lambda}\right\}_{\lambda>0}$ is relatively compact in the space $L_{l o c}^{1}\left((0, \infty), L^{1}(\mathbb{R})\right)$.

Lemma 3.6. For any initial data $u_{0} \in L^{1}(\mathbb{R}) \cap L^{\infty}(\mathbb{R})$ there exists a positive constant

$$
C=C\left(\left\|u_{0}\right\|_{L^{1}(\mathbb{R})},\left\|u_{0}\right\|_{L^{\infty}(\mathbb{R})}\right)
$$

such that, for any $\lambda \geq 1$ and any $r>0$, the following holds:

$$
\int_{|x|>2 r}\left|u^{\lambda}(t, x)\right| d x \leq \int_{|x|>r}\left|u_{0}(x)\right| d x+C\left(\frac{\sqrt{t}}{r}+\frac{t}{r^{2}}\right)
$$

where $u^{\lambda}$ is the solution of system (3.10) with initial data $u_{0}^{\lambda}(\cdot)=\lambda u_{0}(\lambda \cdot)$.

Proof. In order to prove the above estimate we first remark that it is sufficient to consider positive solutions. The comparison principle given in Lemma 3.5 shows that $v \leq u^{\lambda} \leq w$ where $v$ and $w$ are solutions of (3.10) with initial data $-\left|u_{0}^{\lambda}\right|$ and $\left|u_{0}^{\lambda}\right|$ respectively. For $w$ we need estimates for nonnegative solutions whereas for $v$ we have to observe that $-v$ is the solution of the equation with nonnegative initial data similar to (3.10) but replacing the term $u u_{x}$ by $-u u_{x}$. This change does not affect the estimates in Lemma 3.6. Then $\left|u_{\lambda}\right| \leq \max \{|v|, w\}$. Taking into account this argument, it is enough to prove the desired estimate only for nonnegative solutions.

For every $r>0$, let us define $\rho_{r}(z)=\rho(z / r)$, where $\rho$ is a nonnegative $C^{\infty}$ function such that $0 \leq \rho \leq 1$ and

$$
\rho(z)= \begin{cases}0, & |z|<1, \\ 1, & |z|>2 .\end{cases}
$$

Let us assume that $u_{\lambda}$ is a nonnegative solution. We multiply (3.10) by $\rho_{r}$ and integrate it over $(0, t) \times \mathbb{R}$. We obtain:

$$
\begin{aligned}
\int_{0}^{t} \int_{\mathbb{R}} u_{s}^{\lambda}(s) \rho_{r} d x d s=- & \int_{0}^{t} \psi^{\frac{\Delta t}{\lambda^{2}}}(s) \int_{\mathbb{R}}\left(u^{\lambda}(s)\right)^{2} \rho_{r}^{\prime} d x d s+2 \int_{0}^{t} \psi^{\frac{\Delta t}{\lambda^{2}}}(s) \int_{\mathbb{R}} u^{\lambda}(s) \rho_{r}^{\prime \prime} d x d s \\
& +2 \int_{0}^{t}\left(1-\psi^{\frac{\Delta t}{\lambda^{2}}}(s)\right) \int_{\mathbb{R}}\left(\lambda^{2}\left(K_{\lambda} * u^{\lambda}(s)-u^{\lambda}(s)\right)+\lambda u_{x}^{\lambda}(s)\right) \rho_{r} d x d s
\end{aligned}
$$


and, therefore,

$$
\begin{aligned}
\int_{\mathbb{R}} u^{\lambda}(t) \rho_{r} d x \leq & \int_{\mathbb{R}} u_{0}^{\lambda} \rho_{r} d x+\frac{\left\|\rho^{\prime}\right\|_{L^{\infty}(\mathbb{R})}}{r} \int_{0}^{t}\left\|u^{\lambda}(s)\right\|_{L^{2}(\mathbb{R})}^{2} d s \\
& +\frac{\left\|\rho^{\prime \prime}\right\|_{L^{\infty}(\mathbb{R})}}{r^{2}} \int_{0}^{t}\left\|u^{\lambda}(s)\right\|_{L^{1}(\mathbb{R})} d s \\
& +2 \int_{0}^{t}\left(1-\psi^{\frac{\Delta t}{\lambda^{2}}}(s)\right) \int_{\mathbb{R}}\left(\lambda^{2}\left(K_{\lambda} * u^{\lambda}(s)-u^{\lambda}(s)\right)+\lambda u_{x}^{\lambda}(s)\right) \rho_{r} d x d s .
\end{aligned}
$$

We have to obtain an estimate on the last term, uniformly on $\lambda$. The same argument as in $[16$, Theorem 2.5] shows that

$$
\begin{aligned}
\int_{\mathbb{R}}\left(\lambda^{2}\left(K_{\lambda} * u^{\lambda}(s)-u^{\lambda}(s)\right)+\lambda u_{x}^{\lambda}(s)\right) \rho_{r} d x & \lesssim\left\|u_{0}\right\|_{L^{1}(\mathbb{R})}\left\|\rho_{r}^{\prime \prime}\right\|_{L^{\infty}(\mathbb{R})} \\
& \lesssim \frac{\left\|u_{0}\right\|_{L^{1}(\mathbb{R})}\left\|\rho^{\prime \prime}\right\|_{L^{\infty}(\mathbb{R})}}{r^{2}} .
\end{aligned}
$$

So, plugging this into (3.12) and using Lemma 3.1, we get:

$$
\int_{\mathbb{R}} u^{\lambda}(t, x) \rho_{r}(x) d x \leq \int_{\mathbb{R}} u_{0}(x) \rho_{\lambda r}(x) d x+C\left(\frac{\sqrt{t}}{r}+\frac{t}{r^{2}}\right),
$$

where constant $C$ depends only on $\left\|u_{0}\right\|_{L^{1}(\mathbb{R})},\left\|u_{0}\right\|_{L^{\infty}(\mathbb{R})}$ and $\|\rho\|_{W^{2, \infty}(\mathbb{R})}$, which are bounded. For $\lambda \geq 1$, using the definition of $\rho_{\lambda r}$ we finally conclude

$$
\int_{|x|>2 r}\left|u^{\lambda}(t, x)\right| d x \leq \int_{|x|>r}\left|u_{0}(x)\right| d x+C\left(\frac{\sqrt{t}}{r}+\frac{t}{r^{2}}\right) .
$$

The proof is now finished.

3.3. Compactness in $L_{l o c}^{1}\left((0, \infty), L^{1}(\mathbb{R})\right)$. We now proceed as in [15]. For any $0<\tau_{1}<\tau_{2}<\infty$, Lemma 3.1 and Lemma 3.4 give us the existence of a function $\bar{u} \in C\left(\left[\tau_{1}, \tau_{2}\right], H^{-\varepsilon}(\mathbb{R})\right)$, with $\varepsilon \in(0,1)$, such that, up to a subsequence that we do not relabel,

$$
u^{\lambda} \rightarrow \bar{u} \quad \text { in } \quad C\left(\left[\tau_{1}, \tau_{2}\right], H^{-\varepsilon}(\mathbb{R})\right) .
$$

Moreover, for every $t>0$ and $p \in[1, \infty)$, we have that

$$
u^{\lambda}(t) \rightarrow \bar{u}(t) \quad \text { in } \quad L_{l o c}^{p}(\mathbb{R}) .
$$

In fact, the bound from Lemma 3.1 transfers to $\bar{u}$ and, hence,

$$
\|\bar{u}(t)\|_{L^{p}(\mathbb{R})} \leq \liminf _{\lambda \rightarrow \infty}\left\|u^{\lambda}(t)\right\|_{L^{p}(\mathbb{R})} \leq C t^{-\frac{1}{2}\left(1-\frac{1}{p}\right)}, \quad \forall t>0 .
$$

Now, from Lemma 3.1, Lemma 3.4 and Lemma 3.6 we deduce that, for any $0<\tau_{1}<\tau_{2}<\infty$, there exists a positive constant $C=C\left(\tau_{1},\left\|u_{0}\right\|_{L^{1}(\mathbb{R})},\left\|u_{0}\right\|_{L^{\infty}(\mathbb{R})}\right)$ such that

$$
\begin{gathered}
\left\|u^{\lambda}(t)\right\|_{L^{2}\left(\left(\tau_{1}, \tau_{2}\right) \times \mathbb{R}\right)} \leq C, \\
\lambda^{2} \int_{\tau_{1}}^{\tau_{2}} \int_{\mathbb{R}} \int_{\mathbb{R}} J_{\lambda}(x-y)\left(u^{\lambda}(t, x)-u^{\lambda}(t, y)\right)^{2} d x d y d t \leq C,
\end{gathered}
$$

and

$$
\left\|u_{t}^{\lambda}\right\|_{L^{2}\left(\left(\tau_{1}, \tau_{2}\right), H^{-1}(\mathbb{R})\right)} \leq C .
$$


Thus, we can apply Theorem 3.1 to the family $\left\{u^{\lambda}\right\}_{\lambda>0}$ and to the time interval $\left(\tau_{1}, \tau_{2}\right)$. We obtain that there exists a function $\omega \in L^{2}\left(\left(\tau_{1}, \tau_{2}\right), H^{1}(\mathbb{R})\right)$ such that, up to a subsequence,

$$
u^{\lambda} \rightarrow \omega \quad \text { in } L^{2}\left(\left(\tau_{1}, \tau_{2}\right), L_{l o c}^{2}(\mathbb{R})\right)
$$

In fact, due to the previous analysis, we know that $\omega=\bar{u}$. Therefore, we conclude that

$$
\bar{u} \in L^{2}\left(\left(\tau_{1}, \tau_{2}\right), H^{1}(\mathbb{R})\right) \cap L_{l o c}^{1}\left(\left(\tau_{1}, \tau_{2}\right) \times \mathbb{R}\right)
$$

and that

$$
u^{\lambda} \rightarrow \bar{u} \quad \text { in } \quad L_{l o c}^{1}((0, \infty) \times \mathbb{R}) .
$$

Finally, the strong convergence in $L_{\text {loc }}^{1}\left((0, \infty), L^{1}(\mathbb{R})\right)$ is an immediate consequence of Lemma 3.6. Indeed, a standard diagonal argument reduces the proof of the compactness in $L_{l o c}^{1}\left((0, \infty), L^{1}(\mathbb{R})\right)$ to show that

$$
\int_{\tau_{1}}^{\tau_{2}}\left\|u^{\lambda}(t)\right\|_{L^{1}(|x|>r)} d t \rightarrow 0 \quad \text { as } \quad r \rightarrow \infty, \quad \text { uniformly in } \lambda \geq 1,
$$

a property that follows from Lemma 3.6.

3.4. Proof of Theorem 1.2. We can now prove the last main result of this paper. We proceed in several steps, as in [15].

Step I. Passing to the limit. Let us multiply equation (3.10) by a test function $\varphi \in C_{c}^{\infty}(\mathbb{R})$ and integrate it on $(\tau, t) \times \mathbb{R}$, where $0<\tau<t$. We have:

$$
\begin{aligned}
\int_{\mathbb{R}} u^{\lambda}(t, x) \varphi(x) d x-\int_{\mathbb{R}} u^{\lambda}(\tau, x) \varphi(x) d x \\
=2 \int_{\tau}^{t} \psi^{\frac{\Delta t}{\lambda^{2}}}(s) \int_{\mathbb{R}} u_{x x}^{\lambda}(s, x) \varphi(x) d x d s+2 \int_{\tau}^{t} \psi^{\frac{\Delta t}{\lambda^{2}}}(s) \int_{\mathbb{R}} u^{\lambda}(s, x) u_{x}^{\lambda}(s, x) \varphi(x) d x d s \\
\quad+2 \int_{\tau}^{t}\left(1-\psi^{\frac{\Delta t}{\lambda^{2}}}(s)\right) \int_{\mathbb{R}}\left(\lambda^{2} K_{\lambda} * u^{\lambda}(s, x)-\lambda^{2} u^{\lambda}(s, x)+\lambda u_{x}^{\lambda}(s, x)\right) \varphi(x) d x d s \\
=2 \int_{\tau}^{t} \psi^{\frac{\Delta t}{\lambda^{2}}}(s) \int_{\mathbb{R}} u^{\lambda}(s, x) \varphi^{\prime \prime}(x) d x d s-\int_{\tau}^{t} \psi^{\frac{\Delta t}{\lambda^{2}}}(s) \int_{\mathbb{R}}\left(u^{\lambda}(s, x)\right)^{2} \varphi^{\prime}(x) d x d s \\
\quad+2 \int_{\tau}^{t}\left(1-\psi^{\frac{\Delta t}{\lambda^{2}}}(s)\right) \int_{\mathbb{R}} u^{\lambda}(s, x)\left(\lambda^{2} \tilde{K}_{\lambda} * \varphi(x)-\lambda^{2} \varphi(x)-\lambda \varphi^{\prime}(x)\right) d x d s \\
=I_{1}+I_{2}+I_{3},
\end{aligned}
$$

where $\tilde{K}_{\lambda}(z)=K_{\lambda}(-z)$. We know that $u^{\lambda}(t) \rightarrow \bar{u}(t)$ in $L_{l o c}^{p}(\mathbb{R}), 1 \leq p<\infty$, for all $t>0$. Thus,

$$
\int_{\mathbb{R}} u^{\lambda}(t, x) \varphi(x) d x-\int_{\mathbb{R}} u^{\lambda}(\tau, x) \varphi(x) d x \rightarrow \int_{\mathbb{R}} \bar{u}(t, x) \varphi(x) d x-\int_{\mathbb{R}} \bar{u}(\tau, x) \varphi(x) d x .
$$

Let us recall that $\psi^{\Delta t / \lambda^{2}} \rightarrow \frac{1}{2}$ weakly-* in $L^{\infty}((0, \infty))$ as $\Delta t / \lambda^{2} \rightarrow 0$ (see [10], for example). Using that $u^{\lambda} \rightarrow \bar{u}$ in $L_{l o c}^{1}\left((0, \infty), L^{1}(\mathbb{R})\right)$ we obtain that

$$
I_{1} \rightarrow \int_{\tau}^{t} \int_{\mathbb{R}} \bar{u}(s, x) \varphi^{\prime \prime}(x) d x d s, \text { as } \lambda \rightarrow \infty .
$$

The strong convergence of $u^{\lambda}$ towards $\bar{u}$ in $L_{l o c}^{2}((0, \infty) \times \mathbb{R})$ implies that $\left(u^{\lambda}\right)^{2} \rightarrow \bar{u}^{2}$ in $L_{l o c}^{1}((0, \infty) \times$ $\mathbb{R})$ and then

$$
I_{2} \rightarrow \frac{1}{2} \int_{\tau}^{t} \int_{\mathbb{R}}(\bar{u}(s, x))^{2} \varphi^{\prime}(x) d x d s, \text { as } \lambda \rightarrow \infty .
$$


Using the strong convergence of $u^{\lambda}$ in $L_{l o c}^{1}((0, \infty) \times \mathbb{R})$ and that, for any smooth function $\varphi$, we have the following estimate

$$
\left\|\lambda^{2} \tilde{K}_{\lambda} * \varphi(x)-\lambda^{2} \varphi(x)-\lambda \varphi^{\prime}(x)-\varphi^{\prime \prime}\right\|_{L^{\infty}(\mathbb{R})} \lesssim \frac{\left\|\varphi^{\prime \prime \prime}\right\|_{L^{\infty}(\mathbb{R})}}{\lambda}
$$

we obtain that

$$
I_{3} \rightarrow \int_{\tau}^{t} \int_{\mathbb{R}} \bar{u}(s, x) \varphi^{\prime \prime}(x) d x d s, \text { as } \lambda \rightarrow \infty .
$$

In conclusion, all the above convergences show that $\bar{u}$ satisfies

$$
\begin{aligned}
\int_{\mathbb{R}} \bar{u}(t, x) & \varphi(x) d x-\int_{\mathbb{R}} \bar{u}(\tau, x) \varphi(x) d x \\
& =2 \int_{\tau}^{t} \int_{\mathbb{R}} \bar{u}(s, x) \varphi^{\prime \prime}(x) d x d s-\frac{1}{2} \int_{\tau}^{t} \int_{\mathbb{R}}(\bar{u}(s, x))^{2} \varphi^{\prime}(x) d x d s .
\end{aligned}
$$

Note that this implies that $\bar{u}$ is a weak solution of $u_{t}=u u_{x}+2 u_{x x}$.

Step II. Identification of the initial data. Let us choose $\tau=0$ in the previous step. Then, for any $\varphi \in C_{b}^{2}(\mathbb{R})$, we have

$$
\begin{aligned}
& \left|\int_{\mathbb{R}} u^{\lambda}(t, x) \varphi(x) d x-\int_{\mathbb{R}} u^{\lambda}(0, x) \varphi(x) d x\right| \\
& \leq 2\left|\int_{0}^{t} \psi^{\frac{\Delta t}{\lambda^{2}}}(s) \int_{\mathbb{R}} u^{\lambda}(s, x) \varphi^{\prime \prime}(x) d x d s\right|+\left|\int_{0}^{t} \psi^{\frac{\Delta t}{\lambda^{2}}}(s) \int_{\mathbb{R}}\left(u^{\lambda}(s, x)\right)^{2} \varphi^{\prime}(x) d x d s\right| \\
& \quad+2\left|\int_{0}^{t}\left(1-\psi^{\frac{\Delta t}{\lambda^{2}}}(s)\right) \int_{\mathbb{R}} u^{\lambda}(s, x)\left(\lambda^{2} \tilde{K}_{\lambda} * \varphi(x)-\lambda^{2} \varphi(x)-\lambda \varphi^{\prime}(x)\right) d x d s\right| \\
& \quad \lesssim\left\|\varphi^{\prime \prime}\right\|_{L^{\infty}(\mathbb{R})} \int_{0}^{t}\left\|u^{\lambda}(s)\right\|_{L^{1}(\mathbb{R})} d s+\left\|\varphi^{\prime}\right\|_{L^{\infty}(\mathbb{R})} \int_{0}^{t}\left\|u^{\lambda}(s)\right\|_{L^{2}(\mathbb{R})}^{2} d s,
\end{aligned}
$$

where we used that (see [15] for similar estimates) there exists a positive constant $C$ such that, for any $\lambda>0$ and $\varphi \in C_{b}^{2}(\mathbb{R})$, the following holds

$$
\left\|\lambda^{2}\left(K_{\lambda} * \varphi-\varphi\right)-\lambda \varphi^{\prime}\right\|_{L^{\infty}(\mathbb{R})} \leq C\left\|\varphi^{\prime \prime}\right\|_{L^{\infty}(\mathbb{R})} .
$$

Using Lemma 3.2, we deduce that

$$
\left|\int_{\mathbb{R}} u^{\lambda}(t, x) \varphi(x) d x-\int_{\mathbb{R}} u^{\lambda}(0, x) \varphi(x) d x\right| \lesssim\|\varphi\|_{C_{b}^{2}(\mathbb{R})}\left(t+t^{\frac{1}{2}}\right) .
$$

Letting $\lambda \rightarrow \infty$, we obtain that, for any $\varphi \in C_{b}^{2}(\mathbb{R})$,

$$
\left|\int_{\mathbb{R}} \bar{u}(t, x) \varphi(x) d x-M \varphi(0)\right| \lesssim\|\varphi\|_{C_{b}^{2}(\mathbb{R})}\left(t+t^{\frac{1}{2}}\right),
$$

where $M=\int_{\mathbb{R}} u_{0} d x$ is the mass of the initial data $u_{0}$. This implies that, for any $\varphi \in C_{b}^{2}(\mathbb{R})$, we have

$$
\lim _{t \rightarrow 0} \int_{\mathbb{R}} \bar{u}(t, x) \varphi(x) d x=M \varphi(0) .
$$

Standard density arguments and the tail control in Lemma 3.6 show that the above limit holds for any bounded continuous function $\varphi$. We can conclude that $\bar{u}(t)$ goes to $M \delta_{0}$ as $t \rightarrow 0$ in the sense 
of bounded measures. Therefore, we have proved that $\bar{u} \in L_{l o c}^{\infty}\left((0, \infty), L^{1}(\mathbb{R})\right) \cap L_{l o c}^{2}\left((0, \infty), H^{1}(\mathbb{R})\right)$ is the unique solution $u_{M}$ of the following viscous Burgers equation:

$$
\begin{cases}u_{t}=u u_{x}+2 u_{x x}, & (t, x) \in(0, \infty) \times \mathbb{R}, \\ u(0, x)=M \delta_{0}, & x \in \mathbb{R} .\end{cases}
$$

Since this equation has a unique solution, then the whole sequence $u^{\lambda}$ converges to $\bar{u}$, not only a subsequence.

Step III. Asymptotic behavior. The strong convergence of $u^{\lambda}$ toward $u_{M}$ in $L_{l o c}^{1}\left((0, \infty), L^{1}(\mathbb{R})\right)$ also guarantees that for a.e. $t>0$ we have

$$
\left\|u^{\lambda}(t)-u_{M}(t)\right\|_{L^{1}(\mathbb{R})} \rightarrow 0 \quad \text { as } \quad \lambda \rightarrow \infty .
$$

In particular, there exists some $t_{0}>0$ such that

$$
\left\|u^{\lambda}\left(t_{0}\right)-u_{M}\left(t_{0}\right)\right\|_{L^{1}(\mathbb{R})} \rightarrow 0 \quad \text { as } \quad \lambda \rightarrow \infty .
$$

If we set $t=\lambda^{2} t_{0}$ and use the self-similar form of $u_{M}$, we obtain

$$
\lim _{t \rightarrow \infty}\left\|u(t)-u_{M}(t)\right\|_{L^{1}(\mathbb{R})}=0 .
$$

This is exactly the case $p=1$ of (1.8). The general case $p \in[1, \infty)$ follows immediately, since

$$
\begin{aligned}
\left\|u(t)-u_{M}(t)\right\|_{L^{p}(\mathbb{R})} & \lesssim\left\|u(t)-u_{M}(t)\right\|_{L^{1}(\mathbb{R})}^{\frac{1}{2 p-1}}\left(\|u(t)\|_{L^{2 p}(\mathbb{R})}+\left\|u_{M}(t)\right\|_{L^{2 p}(\mathbb{R})}\right)^{1-\frac{1}{2 p-1}} \\
& \lesssim\left\|u(t)-u_{M}(t)\right\|_{L^{1}(\mathbb{R})}^{\frac{1}{2 p-1}}\left(t^{-\frac{1}{2}\left(1-\frac{1}{2 p}\right)}\right)^{1-\frac{1}{2 p-1}}=o\left(t^{-\frac{1}{2}\left(1-\frac{1}{p}\right)}\right) .
\end{aligned}
$$

The proof of Theorem 1.2 is now complete.

\section{NUMERICAL EXAMPLE}

To conclude this paper, we show some numerical simulations that illustrate the analytical results that we have proved in Section 2 and Section 3. Let us remark that the use of splitting methods facilitates the numerical resolution of the different terms of an equation independently. In our case, one can deal with the nonlocal term more efficiently while using appropriate schemes for the nonlinear phenomena arising due to the flux term.

The solution to the augmented Burgers equation, defined as in (1.1), is immediately dominated by the viscous effects of the terms in the right-hand side. In order to emphasize the effects of the nonlinear flux, which are predominant in the sonic-boom phenomenon, let us take some non-unitary values of the parameters in equation (1.1) to perform the simulations:

$$
u_{t}-\left(\frac{u^{2}}{2}\right)_{x}=\frac{1}{\Gamma} u_{x x}+c_{v}\left(K * u-u+u_{x}\right) .
$$

In particular, let us consider $\Gamma=100$ and $c_{V}=0.02$ in our experiments. Note that the analytical results obtained in the previous sections, regarding the convergence order and the decay rates, are still satisfied.

For some grid parameters $\Delta x, \Delta t>0$, let us denote by $u_{j}^{n}$ the approximate solution to $u(n \Delta t, j \Delta x)$, with $n \in \mathbb{N} \cup\{0\}$ and $j \in \mathbb{Z}$. On the one hand, in order to solve equation (1.4), we use Engquist-Osher 
numerical flux and centered finite differences for the diffusion:

$$
u_{j}^{n+\frac{1}{2}}=u_{n}^{j}-\frac{\Delta t}{\Delta x}\left(g\left(u_{j}^{n}, u_{j+1}^{n}\right)-g\left(u_{j-1}^{n}, u_{j}^{n}\right)\right)+\frac{1}{\Gamma} \frac{\Delta t}{\Delta x^{2}}\left(u_{j-1}^{n}-2 u_{j}^{n}+u_{j+1}^{n}\right),
$$

where

$$
g(a, b)=-\frac{a(a-|a|)}{4}-\frac{b(b+|b|)}{4}, \quad \forall a, b \in \mathbb{R} .
$$

This choice for the flux is in accordance with the large-time performance requirements listed in [16, 17].

On the other hand, regarding the equation corresponding to the nonlocal term (1.3), note that it can be rewritten in the following way:

$$
\begin{cases}v_{t}+v_{t x}=c_{v} v_{x x}, & (t, x) \in(0, \infty) \times \mathbb{R}, \\ v(0, x)=v_{0}(x), & x \in \mathbb{R}\end{cases}
$$

This form allows us to use centered finite differences along with Crank-Nicolson (e.g. [21]):

$$
\begin{aligned}
\frac{u_{j}^{n+1}-u_{j}^{n+\frac{1}{2}}}{\Delta t} & +\left(\frac{u_{j+1}^{n+1}-u_{j+1}^{n+\frac{1}{2}}}{\Delta x \Delta t}-\frac{u_{j-1}^{n+1}-u_{j-1}^{n+\frac{1}{2}}}{\Delta x \Delta t}\right) \\
& =\frac{c_{v}}{2}\left(\frac{u_{j-1}^{n+1}-2 u_{j}^{n+1}+u_{j+1}^{n+1}}{\Delta x^{2}}+\frac{u_{j-1}^{n+\frac{1}{2}}-2 u_{j}^{n+\frac{1}{2}}+u_{j+1}^{n+\frac{1}{2}}}{\Delta x^{2}}\right), j \in \mathbb{Z}, n>0 .
\end{aligned}
$$

This alternative has already been used in [9, 23, 24]. Any tridiagonal matrix solver can efficiently solve it, avoiding the computational cost of the integration of the convolution in (1.3). Additionally, simulations show that, combined with (1.4), the long-time dynamics of (1.1) are preserved, while the solution keeps the scale given by (3.9).

We take a mesh size $\Delta x=0.1$ and a time step small enough to satisfy the stability condition (e.g., see [7]) for scheme (4.1), given by:

$$
\left(\max _{j \in \mathbb{Z}}\left|u_{j}^{0}\right|\right)^{2} \frac{\Delta t}{\Delta x}+\frac{2}{\Gamma} \frac{\Delta t}{\Delta x^{2}} \leq 1 .
$$

In order to avoid boundary issues, we choose compactly supported initial data and a large enough spatial domain.

First of all, we focus on the convergence order of the splitting method. Since we do not know the exact solution of (1.1), we determine the order by comparing two numerical solutions for the same initial data but a different time step. That is, we compute $\left\|u_{1}(T)-u_{2}(T)\right\|_{L^{2}(\mathbb{R})}$, where $u_{1}$ and $u_{2}$ are obtained from the same initial data using $\Delta t$ and $\Delta t / 2$ time-steps, respectively. As a matter of fact, we show the convergence order for two different initial data, plotted in Figure 1.

As it can be observed in Figure 2, the slopes for the numerical solutions at $T=10$ corresponding to those initial data are consistent with the theoretical results of Section 2. A line of slope one has been added as a reference for the convergence order.

Furthermore, we also show the convergence rate of the numerical solutions towards the asymptotic profile of the augmented Burgers equation, given in Theorem 1.2. Let us remark that $u_{M}$ is now the asymptotic profile of the viscous Burgers equation with $1 / \Gamma+c_{V}$ viscosity coefficient instead of 2 (see [16] for the complete expression for $u_{M}$ ). We compare the splitting method described above with 

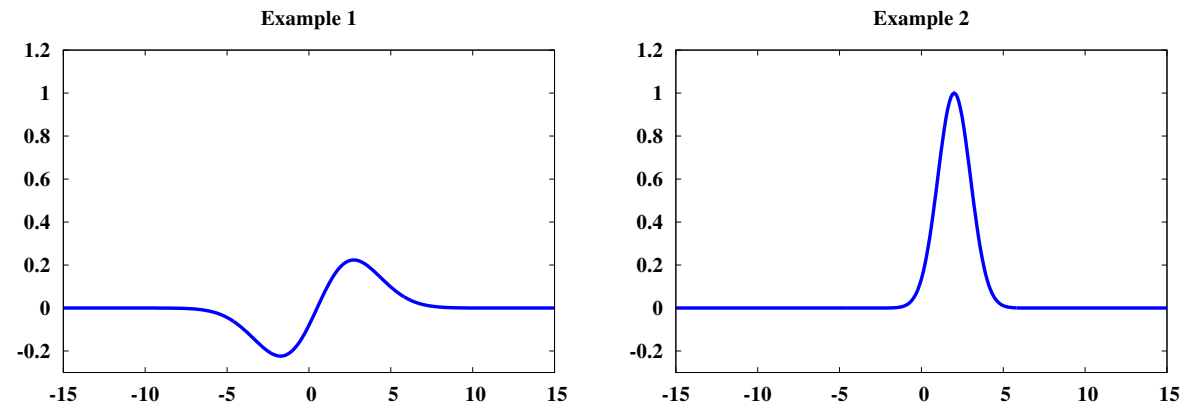

FIGURE 1. Initial data used in the first experiment, in relation to the accuracy order.

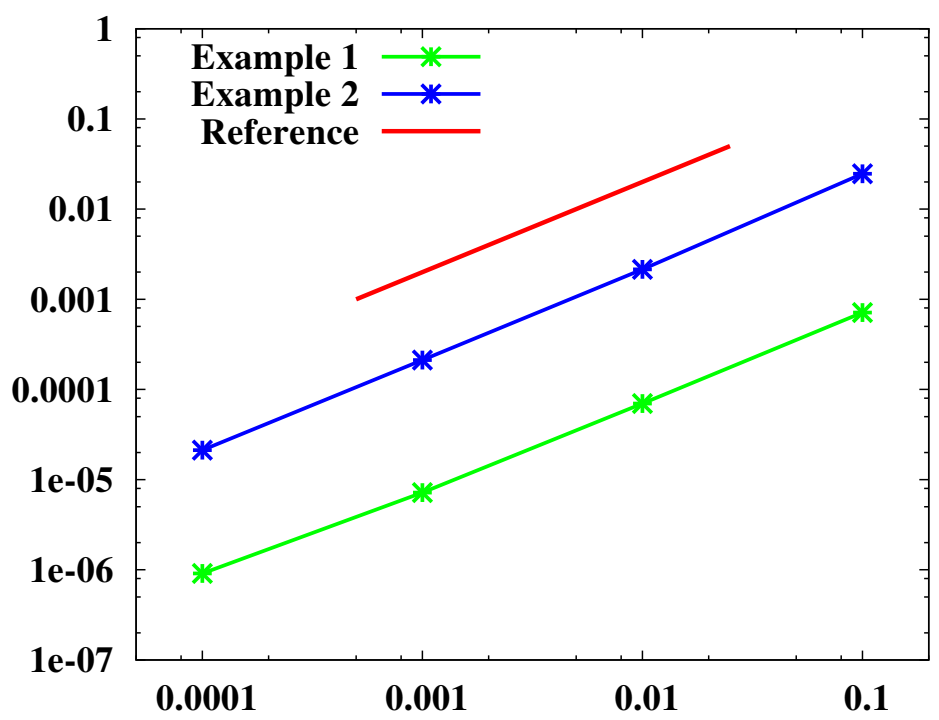

FIgURE 2. Accuracy order for the initial data shown in Figure 1 using several timestep sizes. The plot shows $\left\|u_{1}(10)-u_{2}(10)\right\|_{L^{2}(\mathbb{R})}$, where $u_{1}$ and $u_{2}$ have been computed using $\Delta t$ and $\Delta t / 2$ respectively.

the discretization proposed in [16], where a rectangle method is used to approximate the convolution in (1.1). In Figure 3 we plot the solution at time $T=12000$ for the given initial data, as well as the corresponding asymptotic profile $u_{M}$. Then, in Figure 4 we can observe that the convergence rates meet the ones defined in (1.8), preserving the large-time behavior of the solution of equation (1.1).

\section{ACKNOWLEDGEMENTS}

This work began during the visit of A.P. to IMAR and was finished during the visit of L.I. to BCAM. The authors thank these centers for the hospitality during their visits. 

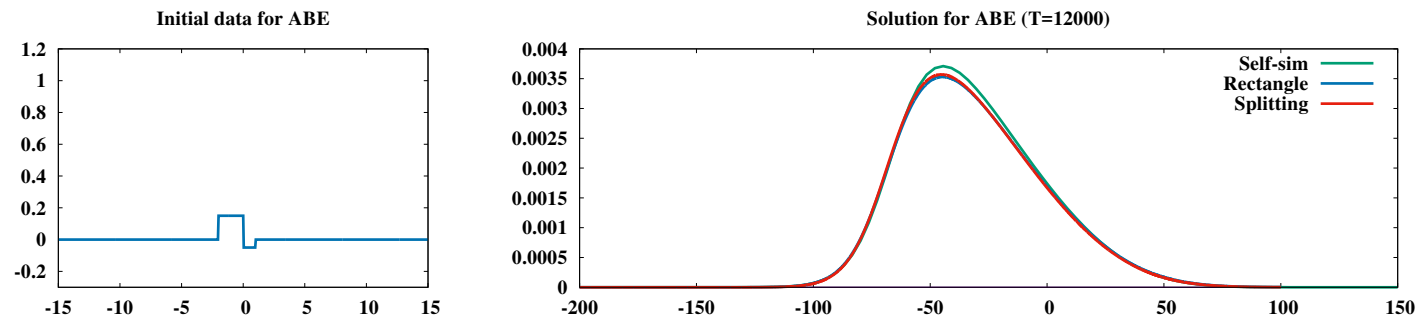

FIgURE 3. Initial data and solution for $\mathrm{ABE}$ with $\Gamma=100$ and $c_{V}=0.02$ at $T=$ 12000. We compare the splitting method (red) with the one from [16] discretized explicitly in time (blue). The self-similar asymptotic profile (green) is given as reference too.
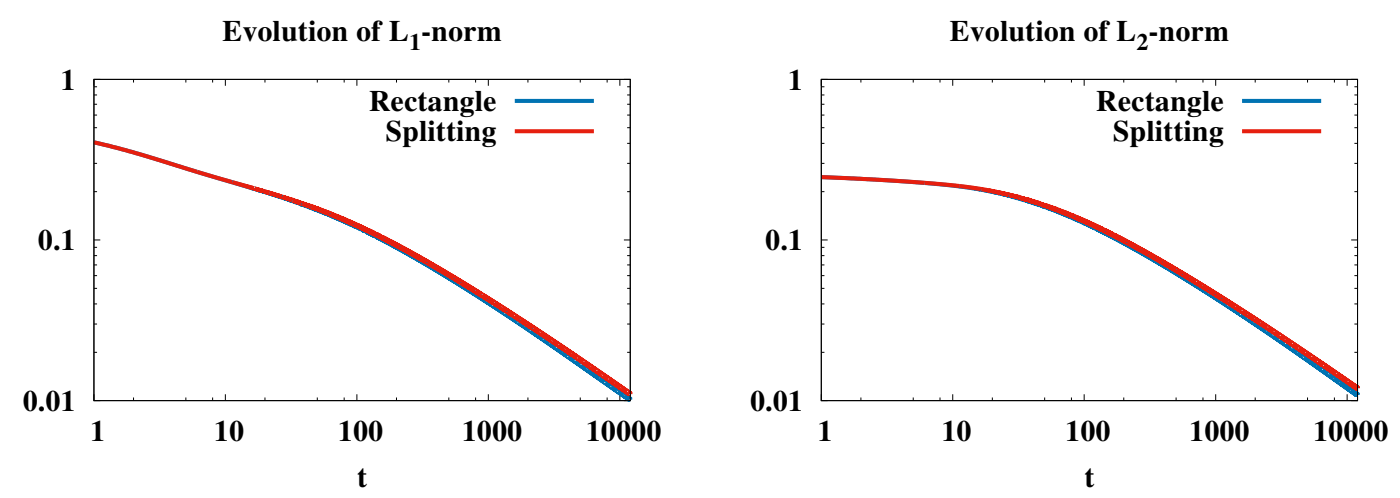

FIGURE 4. Evolution of the $L^{1}$ and $L^{2}$ norms of the difference between the asymptotic profile and the solution, multiplied by their corresponding rate, as in (1.8). We compare the splitting method (red) and the one from [16] discretized explicitly in time (blue).

\section{REFERENCES}

1. J. Aguirre, M. Escobedo, and E. Zuazua, Self-similar solutions of a convection diffusion equation and related semilinear elliptic problems, Communications in Partial Differential Equations 15 (1990), no. 2, 139-157.

2. Juan J. Alonso and Michael R. Colonno, Multidisciplinary optimization with applications to sonic-boom minimization, Annual Review of Fluid Mechanics 44 (2012), 505-526.

3. Piotr Biler, Tadahisa Funaki, and Wojbor A. Woyczyński, Fractal Burgers equations, Journal of Differential Equations 148 (1998), no. 1, 9-46.

4. Piotr Biler, Grzegorz Karch, and Wojbor A. Woyczyński, Asymptotics for conservation laws involving Lévy diffusion generators, Studia Mathematica 148 (2001), no. 2, 171-192.

5. __ Critical nonlinearity exponent and self-similar asymptotics for Lévy conservation laws, Annales de l'Institut Henri Poincare (C) Non Linear Analysis 18 (2001), no. 5, 613-637.

6. Afaf Bouharguane and Rémi Carles, Splitting methods for the nonlocal fowler equation, Mathematics of Computation 83 (2013), 1221-1141.

7. Carlos Castro, Francisco Palacios, and Enrique Zuazua, Optimal control and vanishing viscosity for the Burgers equation, Integral Methods in Science and Engineering (C. Costanda and M. E. Pérez, eds.), vol. 2, Birkhäuser Verlag, 2010, pp. 65-90.

8. Thierry Cazenave and Alain Haraux, An introduction to semilinear evolution equations, Oxford Lecture Series in Mathematics and its Applications, vol. 13, Oxford Univeristy Press, 1998. 
9. Robin Olev Cleveland, Propagation of sonic booms through a real, stratified atmosphere, Ph.D. thesis, University of Texas at Austin, 1995.

10. Michael G. Crandall and Andrew Majda, The method of fractional steps for conservation laws, Numerische Mathematik 34 (1980), 285-314.

11. Constantine M. Dafermos, Hyperbolic conservation laws in continuum physics, Grundlehren der mathematischen Wissenschaften, vol. 325, Springer-Verlag, 2010.

12. Miguel Escobedo and Enrique Zuazua, Large time behavior for convection-diffusion equations in $\mathbb{R}^{n}$, Journal of Functional Analysis 100 (1991), no. 1, 119-161.

13. Helge Holden, Kenneth H. Karlsen, Knut-Andreas Lie, and Nils Henrik Risebro, Splitting methods for partial differential equations with rough solutions, EMS Series of Lectures in Mathematics, European Mathematical Society, 2010.

14. Helge Holden, Christian Lubich, and Nils Henrik Risebro, Operator splitting for partial differential equations with burgers nonlinearity, Mathematics of Computation 82 (2013), no. 281, 173-185.

15. Liviu I. Ignat, Tatiana I. Ignat, and Denisa Stancu-Dumitru, A compactness tool for the analysis of nonlocal evolution equations, SIAM Journal on Mathematical Analysis 47 (2015), no. 2, 1330-1354.

16. Liviu I. Ignat and Alejandro Pozo, A semi-discrete large-time behavior preserving scheme for the augmented Burgers equation, Accepted to M2AN, https://arxiv.org/abs/1409.3817 (2017).

17. Liviu I. Ignat, Alejandro Pozo, and Enrique Zuazua, Large-time asymptotics, vanishing viscosity and numerics for 1-d scalar conservation laws, Mathematics of Computation 84 (2015), 1633-1662.

18. Liviu I. Ignat and Julio D. Rossi, A nonlocal convection-diffusion equation, Journal of Functional Analysis 251 (2007), 399-437.

19. __ Decay estimates for nonlocal problems via energy methods, Journal de Mathématiques Pures et Appliquées 92 (2009), no. 2, 163-187.

20. Liviu I. Ignat and Diana Stan, Asymptotic behaviour for fractional diffusion-convection equations, https://arxiv.org/abs/1703.02908 (2017).

21. Arieh Iserles, A first course in the numerical analysis of differential equations, Cambridge University Press, 1996.

22. Philippe Laurençot, Asymptotic self-similarity for a simplified model for radiating gases, Asymptotic Analysis 42 (2005), 251-262.

23. Sriram K. Rallabhandi, Advanced sonic boom prediction using augmented Burger's equation, Journal of Aircraft 48 (2011), no. 4, 1245-1253.

24. __ Sonic boom adjoint methodology and its applications, 29th AIAA Applied Aerodynamics Conference, American Institute of Aeronautics and Astronautics, 2011.

LIVIU I. IGNAT

InSTitute OF Mathematics “Simion Stollow” OF THE Romanian ACADEMy,

21 Calea Grivitei Street, 010702 Bucharest, Romania.

E-mail address: liviu.ignat@gmail.com

AleJANDRo POZO

INNOVALIA ASSOCIATION,

Carretera de Asua 6, E-48930 Las Arenas - Getxo, Basque Country - Spain.

AND

BCAM - BASQUe CENTER For APPlied MATHEMATiCs,

Alameda de Mazarredo 14, E-48009 Bilbao, Basque Country - Spain.

E-mail address: alejandropozo@gmail.com 